\title{
Small-step and big-step semantics for call-by-need
}

\author{
KEIKO NAKATA \\ Institute of Cybernetics, Tallinn University of Technology, Tallinn, Estonia \\ (e-mail: keiko@cs.ioc.ee) \\ MASAHITO HASEGAWA \\ Research Institute for Mathematical Sciences, Kyoto University, Kyoto, Japan \\ (e-mail: hassei@kurims.kyoto-u.ac.jp)
}

\begin{abstract}
We present natural semantics for acyclic as well as cyclic call-by-need lambda calculi, which are proved equivalent to the reduction semantics given by Ariola and Felleisen (J. Funct. Program., vol. 7, no. 3, 1997). The natural semantics are big-step and use global heaps, where evaluation is suspended and memorized. The reduction semantics are small-step, and evaluation is suspended and memorized locally in let-bindings. Thus two styles of formalization describe the call-by-need strategy from different angles. The natural semantics for the acyclic calculus is revised from the previous presentation by Maraist et al. (J. Funct. Program., vol. 8, no. 3, 1998), and its adequacy is ascribed to its correspondence with the reduction semantics, which has been proved equivalent to call-by-name by Ariola and Felleisen. The natural semantics for the cyclic calculus is inspired by that of Launchbury (1993) and Sestoft (1997), and we state its adequacy using a denotational semantics in the style of Launchbury; adequacy of the reduction semantics for the cyclic calculus is in turn ascribed to its correspondence with the natural semantics.
\end{abstract}

\section{Introduction}

Launchbury (1993) studied a natural semantics for a call-by-need lambda calculus with letrec. He showed the semantics adequate using a denotational semantics. Sestoft (1997) later revised Launchbury's semantics. The revised semantics correctly enforces variable hygiene. Moreover the $\alpha$-renaming strategy of the revised semantics is demonstrated to be suitable in the light of possible implementations with heapbased abstract machines.

Ariola and Felleisen (1997) studied an equational theory for an acyclic (nonrecursive) call-by-need lambda calculus. The calculus admits the standardization theorem, which gives rise to a reduction semantics for the calculus. The call-by-need evaluator, induced by the theory, is proved equivalent to the call-by-name evaluator of Plotkin (1975); as a result, the reduction semantics is shown to be adequate. Ariola and Felleisen also presented a cyclic (recursive) call-by-need lambda calculus 
with letrec; however the cyclic calculus has not been explored. For instance, to the best of our knowledge, it has not been known if the calculus relates to call-by-name or if the standard reduction relation, obtained from the one-step reduction relation and evaluation contexts, is adequate.

The two styles of formalization, namely the natural semantics and the reduction semantics, describe the operational semantics for call-by-need from different angles. The natural semantics is big-step, and evaluation is suspended and memorized in a global heap. The semantics of Sestoft (1997) rigorously preserves binding structure, by performing $\alpha$-renaming when allocating fresh locations in a heap. As he demonstrated by deriving abstract machines from the natural semantics, this approach to variable hygiene has a natural correspondence with possible concrete implementations of call-by-need. The reduction semantics is small-step, and evaluation is suspended and memorized locally in let-bindings. It assumes implicit $\alpha$-conversions. In fact we could think implicit renaming in the reduction semantics is an appropriate approach to variable hygiene, since freshness conditions cannot be checked locally. In other words, the reduction semantics allows for stepwise local reasoning of program behaviour using evaluation contexts.

Our work is motivated to bridge the two styles of formalization, both of which we found interesting. Here are the contributions of the paper:

- We present natural semantics for acyclic and cyclic call-by-need lambda calculi and prove them equivalent to the corresponding reduction semantics given by Ariola and Felleisen (1997). For the acyclic calculus we revise the natural semantics given in Maraist et al. (1998) by correctly enforcing variable hygiene in the style of Sestoft $(1997)^{1}$; its adequacy is ascribed to its correspondence with the reduction semantics, which has been proved equivalent to call-byname by Ariola and Felleisen. The natural semantics for the cyclic calculus is very much inspired by Sestoft's and hence by Launchbury's; the main difference is that our semantics directly works with the full lambda terms with letrec, whereas Sestoft's works with the 'normalized' lambda terms, where function arguments are only variables, by having a precompilation step.

- We show the natural semantics for the cyclic calculus adequate by adapting Launchbury's denotational argument. As a consequence the reduction semantics for the cyclic calculus is also shown to be adequate thanks to the equivalence of the two semantics; to the best of our knowledge, this fact has not been shown so far.

\section{Call-by-need let calculus $\lambda_{\text {let }}$}

We first study the operational semantics for the acyclic (non-recursive) calculus.

1 In Maraist et al. (1998) equivalence of the natural semantics and reduction semantics is stated. The paper only mentions that the result is proved by simple induction on derivations in the natural semantics, but we did not find it 'simple'. 


$\begin{array}{lll}\text { Expressions } & M, N & :=x|\lambda x \cdot M| M N \mid \text { let } x \text { be } M \text { in } N \\ \text { Values } & V & ::=\lambda x . M \\ \text { Answers } & A & ::=V \mid \text { let } x \text { be } M \text { in } A \\ \text { Contexts } & E & ::=[]|E M| \text { let } x \text { be } M \text { in } E \mid \text { let } x \text { be } E \text { in } E^{\prime}[x] \\ \text { Heaps } & \Psi, \Phi & ::=\varepsilon \mid \Psi, x \mapsto M\end{array}$

Fig. 1. Syntax of $\lambda_{\text {let }}$.

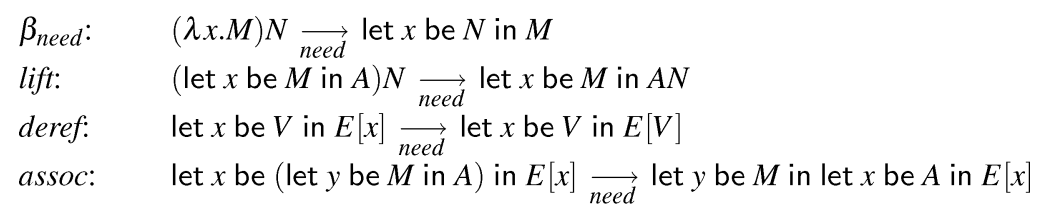

Fig. 2. Reduction semantics for $\lambda_{\text {let }}$.

Lambda
$\langle\Psi\rangle \lambda x . M \Downarrow_{X}\langle\Psi\rangle \lambda x . M$
Application
$\frac{\langle\Psi\rangle M_{1} \Downarrow_{X}\langle\Phi\rangle \lambda x . N \quad\left\langle\Phi, x^{\prime} \mapsto M_{2}\right\rangle N\left[x^{\prime} / x\right] \Downarrow_{X}\left\langle\Psi^{\prime}\right\rangle V \quad x^{\prime} \text { fresh }}{\langle\Psi\rangle M_{1} M_{2} \Downarrow_{X}\left\langle\Psi^{\prime}\right\rangle V}$
Let
$\frac{\left\langle\Psi, x^{\prime} \mapsto N\right\rangle M\left[x^{\prime} / x\right] \Downarrow_{X}\langle\Phi\rangle V \quad x^{\prime} \text { fresh }}{\langle\Psi\rangle \text { let } x \text { be } N \text { in } M \Downarrow_{X}\langle\Phi\rangle V}$
Variable
$\frac{\left.\left.\langle\Psi\rangle M \Downarrow_{X \cup\{x\} \cup \text { dom }(\Phi)}^{\langle\Psi}\right\rangle \Psi^{\prime}\right\rangle V}{\langle\Psi, x \mapsto M, \Phi\rangle x \Downarrow_{X}\left\langle\Psi^{\prime}, x \mapsto V, \Phi\right\rangle V}$

Fig. 3. Natural semantics for $\lambda_{\text {let }}$.

\subsection{Syntax and semantics}

The syntax of the call-by-need let calculus $\lambda_{\text {let }}$ is defined in Figure 1 . The reduction and natural semantics are given in Figures 2 and 3 respectively. The metavariable $X$ ranges over sets of variables. The notation $\epsilon$ denotes an empty sequence. The notation $\operatorname{dom}(\Psi)$ denotes the domain of $\Psi$, namely $\operatorname{dom}(\epsilon)=\emptyset$ and $\operatorname{dom}\left(x_{1} \mapsto\right.$ $\left.M_{1}, \ldots, x_{n} \mapsto M_{n}\right)=\left\{x_{1}, \ldots, x_{n}\right\}$. The notation $M\left[x^{\prime} / x\right]$ denotes substitution of $x^{\prime}$ for free occurrences of $x$ in $M$. The notion of free variables is standard and is defined in Figure 4. A program is a closed expression. We say an expression $M$ (standard) reduces to $N$, written $M \rightarrow N$ if $M=E\left[M^{\prime}\right]$ and $N=E\left[N^{\prime}\right]$, where $M^{\prime} \underset{\text { need }}{\longrightarrow} N^{\prime}$. We write $M \rightarrow N$ to denote that $M$ reduces to $N$ in zero or more steps, i.e. $\rightarrow$ is the reflexive and transitive closure of $\rightarrow$.

The reduction semantics is identical to the previous presentation by Ariola and Felleisen (1997). It works with $\alpha$-equivalence classes of expressions. We assume all binding occurrences of variables in a canonical representative of a class use pairwise distinct names. In particular, evaluation contexts and reduction rules are defined 


$$
\begin{array}{ll}
F V(x) & =\{x\} \\
F V(\lambda x . M) & =F V(M) \backslash\{x\} \\
F V(M N) & =F V(M) \cup F V(N) \\
F V(\text { let } x \text { be } M \text { in } N) & =F V(M) \cup(F V(N) \backslash\{x\})
\end{array}
$$

Fig. 4. Free variables.

over canonical representatives. Below we recall the reduction semantics briefly. The key rule is $\beta_{\text {need }}$, where application reduces to a let-construct, thus suspending evaluation of the argument. Since deref only substitutes values for variables, $\beta_{\text {need }}$ also ensures that evaluation of an argument is shared among all references to the argument in the function body. The administrative rules lift and assoc extend the scopes of let-bound variables so that values surrounded by let's become available without duplicating reducible expressions. The following lemma states that there exists at most one partitioning of a program into a context and a redex, namely the unique-decomposition property. It is proved by induction on $M$.

\section{Lemma 2.1}

For any program $M$, either $M$ is an answer or there exist a unique context $E$ and a redex $N$ such that $M=E[N]$.

The natural semantics is revised from that of Maraist et al. (1998). It differs from the previous presentation in the following two points: Firstly our semantics enforces variable hygiene correctly in the style of Sestoft (1997) by keeping track of variables which are temporarily deleted from heaps in Variable rule. This way, freshness conditions are locally checkable. Secondly our semantics works with the let-explicit calculus instead of the let-free one and hence has an inference rule for the let-construct; this makes it smooth to extend our study of the acyclic calculus to the cyclic calculus in the next section. As in Maraist et al. (1998) the order of bindings in a heap is significant. That is, re-ordering of bindings in a heap is not allowed. In particular in a heap $x_{1} \mapsto M_{1}, x_{2} \mapsto M_{2}, \ldots, x_{n} \mapsto M_{n}$, an expression $M_{i}$ may contain as free variables only $x_{1}, \ldots, x_{i-1}$. This explains why it is safe to remove the bindings on the right in Variable rule: $\Phi$ is not in the scope of $M$. The natural semantics does not assume implicit $\alpha$-renaming but works with (raw) expressions. We may write \langle\rangle $M$ to denote $\langle\epsilon\rangle M$.

A configuration is a pair $\langle\Psi\rangle M$ of a heap and an expression. A configuration $\left\langle x_{1} \mapsto M_{1}, \ldots, x_{n} \mapsto M_{n}\right\rangle N$ is closed if $F V(N) \subseteq\left\{x_{1}, \ldots, x_{n}\right\}$, and $F V\left(M_{i}\right) \subseteq$ $\left\{x_{1}, \ldots, x_{i-1}\right\}$ for any $i$ in $1, \ldots, n$. Borrowing from Sestoft's nomenclature (Sestoft 1997), we say a configuration $\left\langle x_{1} \mapsto M_{1}, \ldots, x_{n} \mapsto M_{n}\right\rangle N$ is $X$-good if $x_{1}, \ldots, x_{n}$ are pairwise distinctly named and $\left\{x_{1}, \ldots, x_{n}\right\}$ and $X$ are disjoint. The judgement $\langle\Psi\rangle M \Downarrow_{X}\langle\Phi\rangle V$ is promising if $\langle\Psi\rangle M$ is closed and $X$-good.

Since derivations in the natural semantics only allocate fresh variables in a heap and substitute fresh variables for variables in expressions, a derivation of a promising judgment is promising everywhere. The following lemma is proved by induction on the derivation of $\langle\Psi\rangle M \Downarrow_{X}\langle\Phi\rangle V$. 


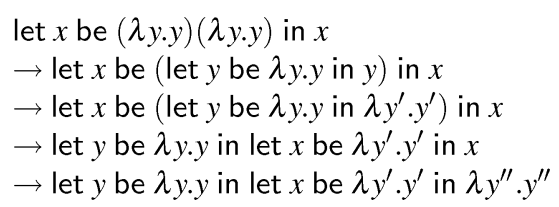

Fig. 5. The reduction sequence for let $x$ be $(\lambda y \cdot y)(\lambda y \cdot y)$ in $x$.

\begin{tabular}{|c|c|}
\hline \multirow[b]{2}{*}{\langle\rangle$\lambda y \cdot y \Downarrow_{\left\{x^{\prime}\right\}}\langle\rangle \lambda y \cdot y$} & \langle\rangle$\lambda y \cdot y \Downarrow_{\left\{x^{\prime}, y^{\prime}\right\}}\langle\rangle \lambda y \cdot y$ \\
\hline & $\overline{\left\langle y^{\prime} \mapsto \lambda y . y\right\rangle y^{\prime} \Downarrow_{\left\{x^{\prime}\right\}}\left\langle y^{\prime} \mapsto \lambda y \cdot y\right\rangle \lambda y \cdot y}$ \\
\hline\langle\rangle$(\lambda y \cdot y)($ & $\lambda y \cdot y) \Downarrow_{\left\{x^{\prime}\right\}}\left\langle y^{\prime} \mapsto \lambda y \cdot y\right\rangle \lambda y \cdot y$ \\
\hline$\left\langle x^{\prime} \mapsto(\lambda y \cdot y)(\lambda y . y\right.$ & )$\rangle x^{\prime} \Downarrow_{\emptyset}\left\langle y^{\prime} \mapsto \lambda y \cdot y, x^{\prime} \mapsto \lambda y \cdot y\right\rangle \lambda y \cdot y$ \\
\hline
\end{tabular}

Fig. 6. The derivation for let $x$ be $(\lambda y \cdot y)(\lambda y \cdot y)$ in $x$.

\section{Lemma 2.2}

If $\langle\Psi\rangle M$ is closed and $X$-good and the judgment $\langle\Psi\rangle M \Downarrow_{X}\langle\Phi\rangle V$ has a derivation, then $\langle\Phi\rangle V$ is closed and $X$-good, and $\operatorname{dom}(\Psi) \subseteq \operatorname{dom}(\Phi)$, and every judgment in the derivation is promising.

Lemma 2.2 shows the natural semantics preserves binding structure in the absence of implicit $\alpha$-renaming. Since the malloc function returns fresh locations in a heap, the natural semantics indeed relates to heap-based implementations of call-by-need.

Example Figures 5 and 6 present the reduction sequence and the derivation for the expression let $x$ be $(\lambda y \cdot y)(\lambda y \cdot y)$ in $x$ respectively.

\subsection{Equivalence of the two semantics}

The idea underlying our proof is derived from observing the following gap between the two semantics:

- In the reduction semantics heaps are first allocated locally and then are globalized as much as necessary by applying lift or assoc afterwards to dereference computed values. Besides, the redex is focused implicitly in the sense that the semantics does not specify how to build evaluation contexts but rather relies on the unique-decomposition property.

- In the natural semantics there is a single global heap. The redex is focused explicitly by applying inference rules, thus decomposing evaluation contexts.

To facilitate reconstructing reduction sequences from derivations by bridging the above gap, our proof introduces an instrumented natural semantics, defined in Figure 7, as an intermediary step. The instrumented natural semantics uses structured heaps $\Sigma$, which are sequences of frames $F$. Intuitively structured heaps are sequenced evaluation contexts. 


$$
\begin{aligned}
& \text { Frames } \quad F \quad::=[] M \mid \text { let } x \text { be } M \text { in [] | let } x \text { be [] in } E[x] \\
& \text { Structured heaps } \quad \Sigma \quad::=\varepsilon \mid \Sigma, F \\
& \text { Let's } \quad \Theta::=\varepsilon \mid \Theta \text {, let } x \text { be } M \text { in [] } \\
& \frac{\operatorname{Lam}}{\vdash\langle\Sigma\rangle \lambda x . M \Downarrow\langle\Sigma\rangle \lambda x \cdot M} \\
& \text { App } \\
& \frac{\vdash\left\langle\Sigma,[] M_{2}\right\rangle M_{1} \Downarrow\left\langle\Sigma_{1},[] M_{2}, \Theta\right\rangle \lambda x . N \quad \vdash\left\langle\Sigma_{1}, \Theta \text {, let } x^{\prime} \text { be } M_{2} \text { in [] }\right] N\left[x^{\prime} / x\right] \Downarrow\left\langle\Sigma_{2}\right\rangle V \quad x^{\prime} \text { fresh }}{\vdash\langle\Sigma\rangle M_{1} M_{2} \Downarrow\left\langle\Sigma_{2}\right\rangle V} \\
& \text { Letin } \\
& \frac{\vdash\left\langle\Sigma \text {, let } x^{\prime} \text { be } N \text { in }[]\right\rangle M\left[x^{\prime} / x\right] \Downarrow\left\langle\Sigma^{\prime}\right\rangle V \quad x^{\prime} \text { fresh }}{\vdash\langle\Sigma\rangle \text { let } x \text { be } N \text { in } M \Downarrow\left\langle\Sigma^{\prime}\right\rangle V} \\
& \text { Var } \\
& \frac{\vdash\left\langle\Sigma \text {, let } x \text { be }[] \text { in } \Sigma_{1}[x]\right\rangle M \Downarrow\left\langle\Sigma_{2} \text {, let } x \text { be [] in } \Sigma_{1}[x], \Theta\right\rangle V}{\vdash\left\langle\Sigma \text {, let } x \text { be } M \text { in [], } \Sigma_{1}\right\rangle x \Downarrow\left\langle\Sigma_{2}, \Theta \text {, let } x \text { be } V \text { in [], } \Sigma_{1}\right\rangle V}
\end{aligned}
$$

Fig. 7. Instrumented natural semantics for $\lambda_{\text {let }}$.

The notation $L B V(\Sigma)$ denotes the set of variables let-bound in frames of $\Sigma$. Or

$$
\begin{aligned}
\operatorname{LBV}(\epsilon) & =\emptyset \\
\operatorname{LBV}(\Sigma,[] M) & =\operatorname{LBV}(\Sigma) \\
\operatorname{LBV}(\Sigma, \text { let } x \text { be } M \text { in }[]) & =\operatorname{LBV}(\Sigma) \cup\{x\} \\
\operatorname{LBV}(\Sigma, \text { let } x \text { be }[] \text { in } M) & =\operatorname{LBV}(\Sigma)
\end{aligned}
$$

A structured heap $\Sigma$ is well-formed if it is an empty sequence, or else $\Sigma=\Sigma^{\prime}, F$, and $\Sigma^{\prime}$ is well-formed and one of the following conditions holds:

(1) $F=[] M$ and $F V(M) \subseteq L B V\left(\Sigma^{\prime}\right)$

(2) $F=$ let $x$ be $M$ in [] and $F V(M) \subseteq L B V\left(\Sigma^{\prime}\right)$ and $x$ is distinct from any of $\operatorname{LBV}\left(\Sigma^{\prime}\right)$

(3) $F=$ let $x$ be [] in $M$ and $F V(M) \subseteq L B V\left(\Sigma^{\prime}\right) \cup\{x\}$ and $x$ is distinct from any of $\operatorname{LBV}\left(\Sigma^{\prime}\right)$

A structured configuration $\langle\Sigma\rangle M$ is well-formed if $\Sigma$ is well-formed and $F V(M) \subseteq$ $\operatorname{LBV}(\Sigma)$.

We map structured configurations to expressions by defining translation $\lfloor\cdot\rfloor$ from structured heaps to evaluation contexts:

$$
\lfloor\epsilon\rfloor=[] \quad\lfloor\Sigma, F\rfloor=\lfloor\Sigma\rfloor[F]
$$

We may identify $\Sigma$ with $\lfloor\Sigma\rfloor$ when there should be no confusion and thus write $\Sigma[M]$ to denote $[\Sigma\rfloor[M]$. A (raw) expression $\Sigma[M]$ is not necessarily a canonical representative of an $\alpha$-equivalence class. The following lemma is proved by induction on the structure of $\Sigma$.

\section{Lemma 2.3}

If $\langle\Sigma\rangle M$ is well-formed, then $\lfloor\Sigma\rfloor[M]$ is a program.

Let's look at the inference rules in Figure 7. Lam and Letin are self-explanatory. When evaluating function expression $M_{1}$ in $A p p$, the rule pushes into the heap 


$$
\begin{aligned}
& \frac{\vdash\left\langle\text { let } x^{\prime} \text { be [] in } x^{\prime} \text {, let } y^{\prime} \text { be [] in } y^{\prime}\right\rangle \lambda y . y \Downarrow\left\langle\text { let } x^{\prime} \text { be }[] \text { in } x^{\prime} \text {, let } y^{\prime} \text { be [] in } y^{\prime}\right\rangle \lambda y . y}{\vdash\left\langle\text { let } x ^ { \prime } \text { be [] in } x ^ { \prime } \text { , let } y ^ { \prime } \text { be } \lambda y \cdot y \text { in [] } y ^ { \prime } \Downarrow \left\langle\text { let } x^{\prime} \text { be [] in } x^{\prime} \text {, let } y^{\prime} \text { be } \lambda y . y \text { in [] } \lambda y . y \quad(*)\right.\right.} \\
& \vdash\left\langle\text { let } x^{\prime} \text { be [] in } x^{\prime},[](\lambda y . y)\right\rangle \lambda y . y \Downarrow\left\langle\text { let } x^{\prime} \text { be [] in } x^{\prime},[](\lambda y . y)\right\rangle \lambda y . y \\
& \vdash\left\langle\text { let } x ^ { \prime } \text { be [] in } x ^ { \prime } \text { , let } y ^ { \prime } \text { be } \lambda y . y \text { in [] } y ^ { \prime } \Downarrow \left\langle\text { let } x^{\prime} \text { be [] in } x^{\prime} \text {, let } y^{\prime} \text { be } \lambda y . y \text { in [] } \lambda y . y \quad(*)\right.\right. \\
& \vdash\left\langle\text { let } x^{\prime} \text { be [] in } x^{\prime}\right\rangle(\lambda y \cdot y)(\lambda y . y) \Downarrow\left\langle\text { let } x^{\prime} \text { be [] in } x^{\prime} \text {, let } y^{\prime} \text { be } \lambda y . y \text { in [] } \lambda y . y\right. \\
& \vdash\left\langle\text { let } x ^ { \prime } \text { be } ( \lambda y . y ) ( \lambda y . y ) \text { in [] } x ^ { \prime } \Downarrow \left\langle\text { let } y^{\prime} \text { be } \lambda y . y \text { in [], let } x^{\prime} \text { be } \lambda y . y \text { in [] } \lambda y . y\right.\right. \\
& \vdash\langle\rangle \text { let } x \text { be }(\lambda y . y)(\lambda y . y) \text { in } x \Downarrow\left\langle\text { let } y^{\prime} \text { be } \lambda y . y \text { in [], let } x^{\prime} \text { be } \lambda y . y \text { in [] } \lambda y . y\right.
\end{aligned}
$$

Fig. 8. The derivation in the instrumented natural semantics for let $x$ be $(\lambda y \cdot y)(\lambda y \cdot y)$ in $x$.

the frame [] $M_{2}$, which is popped when evaluating function body $N$. Notice that the trailing frame to [] $M_{2}$ in the result heap of the left hypothesis is $\Theta$, which suggests $M_{1}$ reduces to an answer $\Theta[\lambda x . N]$. This will be proved in Proposition 2.1. Also, observe the order between $\Theta$ and let $x^{\prime}$ be $M_{2}$ in [] in the right hypothesis, where let-lifting is performed implicitly. When evaluating variable $x$ in Var, the rule pushes the 'continuation' let $x$ be [] in $\Sigma_{1}[x]$ into the heap. Again, observe the order between $\Theta$ and let $x$ be $V$ in [] in the result heap of the consequence, where let-association is implicitly performed. It should be noted that Ariola and Felleisen already observed that Launchbury's formalization has hidden flattening of a heap in his Variable rule, which amounts to applying assoc (Ariola \& Felleisen 1997).

\section{Lemma 2.4}

If $\langle\Sigma\rangle M$ is well-formed and $\vdash\langle\Sigma\rangle M \Downarrow\left\langle\Sigma^{\prime}\right\rangle V$, then $\left\langle\Sigma^{\prime}\right\rangle V$ is well-formed.

\section{Proof}

By induction on the derivation of $\vdash\langle\Sigma\rangle M \Downarrow\left\langle\Sigma^{\prime}\right\rangle V$.

Simple induction proves the instrumented natural semantics correct with respect to the reduction semantics.

\section{Proposition 2.1}

If $\langle\Sigma\rangle M$ is well-formed and $\vdash\langle\Sigma\rangle M \Downarrow\left\langle\Sigma^{\prime}\right\rangle V$, then $\Sigma[M] \rightarrow \Sigma^{\prime}[V]$.

\section{Proof}

By induction on the derivation of $\vdash\langle\Sigma\rangle M \Downarrow\left\langle\Sigma^{\prime}\right\rangle V$ with case analysis on the last rule used.

- The cases of Lam and Letin are obvious.

- The case of App. Suppose we deduce $\vdash\langle\Sigma\rangle M_{1} M_{2} \Downarrow\left\langle\Sigma_{2}\right\rangle V$ from $\vdash$ $\left\langle\Sigma,[] M_{2}\right\rangle M_{1} \Downarrow\left\langle\Sigma_{1},[] M_{2}, \Theta\right\rangle \lambda x . N$ and $\vdash\left\langle\Sigma_{1}, \Theta\right.$, let $x^{\prime}$ be $M_{2}$ in [] $N\left[x^{\prime} / x\right] \Downarrow$ $\left\langle\Sigma_{2}\right\rangle V$. Then we have

$\Sigma\left[M_{1} M_{2}\right]$

$\rightarrow \Sigma_{1}\left[(\Theta[\lambda x . N]) M_{2}\right]$ by ind. hyp.

$\rightarrow \Sigma_{1}\left[\Theta\left[(\lambda x . N) M_{2}\right]\right]$ by lift

$\rightarrow \Sigma_{1}\left[\Theta\left[\right.\right.$ let $x^{\prime}$ be $M_{2}$ in $\left.\left.N\left[x^{\prime} / x\right]\right]\right]$ by $\beta_{\text {need }}$

$\rightarrow \Sigma_{2}[V]$ by ind. hyp.

- The case of Var. Suppose we deduce $\vdash\left\langle\Sigma\right.$, let $x$ be $M$ in [], $\left.\Sigma_{1}\right\rangle x \Downarrow$ $\left\langle\Sigma_{2}, \Theta\right.$, let $x$ be $V$ in [], $\left.\Sigma_{1}\right\rangle \quad V$ from $\vdash\left\langle\Sigma\right.$, let $x$ be [] in $\left.\Sigma_{1}[x]\right\rangle M \Downarrow$ 
$\left\langle\Sigma_{2}\right.$, let $x$ be [] in $\left.\Sigma_{1}[x], \Theta\right\rangle V$. Then we have

$\Sigma$ [let $x$ be $M$ in $\left.\Sigma_{1}[x]\right]$

$\rightarrow \Sigma_{2}\left[\right.$ let $x$ be $\Theta[V]$ in $\left.\Sigma_{1}[x]\right]$ by ind. hyp.

$\rightarrow \Sigma_{2}\left[\Theta\left[\right.\right.$ let $x$ be $V$ in $\left.\left.\Sigma_{1}[x]\right]\right]$ by assoc

$\rightarrow \Sigma_{2}\left[\Theta\left[\right.\right.$ let $x$ be $V$ in $\left.\left.\Sigma_{1}[V]\right]\right]$ by deref

We need to prove the original natural semantics in Figure 3 correct with respect to the instrumented natural semantics. This is mainly to check that in Figure 7 frames are properly pushed and popped so that the pop operation never fails. Below we define a preorder on structured heaps to state that structured heaps only 'grow' during derivations.

A preorder $\leqslant$ on structured heaps is defined such that $F_{1}, \ldots, F_{m} \leqslant F_{1}^{\prime}, \ldots, F_{n}^{\prime}$ if there is an injection $l$ from $\{1, \ldots, m\}$ to $\{1, \ldots, n\}$ satisfying the following three conditions:

(1) if $i<j$, then $\imath(i)<\imath(j)$;

(2) for all $i$ in $\{1, \ldots, m\}$, either $F_{i}=F_{l(i)}^{\prime}$ or else $F_{i}=$ let $x$ be $M$ in [] and $F_{l(i)}^{\prime}=$ let $x$ be $N$ in [] for some $x, M$ and $N$;

(3) for all $i$ in $\{1, \ldots, n\} \backslash \operatorname{ran}(l), F_{i}^{\prime}=$ let $x$ be $M$ in [] for some $x$ and $M$, where $\operatorname{ran}(l)$ denotes the range of $l$ and $\{1, \ldots, n\} \backslash \operatorname{ran}(l)$ denotes set subtraction.

It is easy to check that $\leqslant$ is a preorder.

Lemma 2.5

If $\langle\Sigma\rangle M$ is well-formed and $\vdash\langle\Sigma\rangle M \Downarrow\left\langle\Sigma^{\prime}\right\rangle V$, then $\Sigma \leqslant \Sigma^{\prime}$.

Proof

By induction on the derivation of $\vdash\langle\Sigma\rangle M \Downarrow\left\langle\Sigma^{\prime}\right\rangle V$. We use the fact that if $\Sigma \leqslant \Sigma^{\prime}$ and $\Sigma^{\prime}, \Theta \leqslant \Sigma^{\prime \prime}$, then $\Sigma \leqslant \Sigma^{\prime \prime}$.

We define translation $\lceil\cdot\rceil$ from structured heaps to (ordinary) heaps by collecting let-frames as follows:

$$
\begin{aligned}
\lceil\epsilon\rceil & =\epsilon \\
\lceil\Sigma,[] M\rceil & =\lceil\Sigma\rceil \\
\lceil\Sigma \text {, let } x \text { be } M \text { in }[]\rceil & =\lceil\Sigma\rceil, x \mapsto M \\
\lceil\Sigma, \text { let } x \text { be }[] \text { in } M\rceil & =\lceil\Sigma\rceil
\end{aligned}
$$

Proposition 2.2

If $\langle\Psi\rangle M$ is closed and $X$-good and $\langle\Psi\rangle M \Downarrow_{X}\langle\Phi\rangle V$, then for any $\Sigma$ such that $\lceil\Sigma\rceil=\Psi$ and $\langle\Sigma\rangle M$ is well-formed, $\vdash\langle\Sigma\rangle M \Downarrow\left\langle\Sigma^{\prime}\right\rangle V$ and $\left\lceil\Sigma^{\prime}\right\rceil=\Phi$.

Proof

By induction on the derivation of $\langle\Psi\rangle M \Downarrow_{X}\langle\Phi\rangle V$ with case analysis on the last rule used.

- The cases of Lambda and Let are obvious.

- The case of Application. Suppose $M=M_{1} M_{2}$ and we deduce $\langle\Psi\rangle M_{1} M_{2} \Downarrow_{X}$ $\left\langle\Psi^{\prime}\right\rangle V$ from $\langle\Psi\rangle M_{1} \Downarrow_{X}\langle\Phi\rangle \lambda x . N$ and $\left\langle\Phi, x^{\prime} \mapsto M_{2}\right\rangle N\left[x^{\prime} / x\right] \Downarrow_{X}\left\langle\Psi^{\prime}\right\rangle V$. Suppose $\lceil\Sigma\rceil=\Psi$ and $\langle\Sigma\rangle M_{1} M_{2}$ is well-formed. By ind. hyp. and Lemmas 2.4 
and $2.5, \vdash\left\langle\Sigma,[] M_{2}\right\rangle M_{1} \Downarrow\left\langle\Sigma_{1},[] M_{2}, \Theta\right\rangle \lambda x . N$ and $\left\lceil\Sigma_{1},[] M_{2}, \Theta\right\rceil=\Phi$ and $\left\langle\Sigma_{1},[] M_{2}, \Theta\right\rangle \lambda x . N$ is well-formed. By ind. hyp.,

$\vdash\left\langle\Sigma_{1}\right.$, $\Theta$, let $x^{\prime}$ be $M_{2}$ in []$\rangle N\left[x^{\prime} / x\right] \Downarrow\left\langle\Sigma_{2}\right\rangle V$ and $\left\lceil\Sigma_{2}\right\rceil=\Psi^{\prime}$.

- The case of Variable. Suppose $M=x$ and we deduce $\langle\Psi, x \mapsto N, \Phi\rangle x \Downarrow_{X}$ $\left\langle\Psi^{\prime}, x \mapsto V, \Phi\right\rangle V$ from $\langle\Psi\rangle N \Downarrow_{X \cup\{x\} \cup \operatorname{dom}(\Phi)}\left\langle\Psi^{\prime}\right\rangle V$. Let $\Sigma=\Sigma_{1}$, let $x$ be $N$ in [], $\Sigma_{2}$ with $\left\lceil\Sigma_{1}\right\rceil=\Psi$ and $\left\lceil\Sigma_{2}\right\rceil=\Phi$ and $\langle\Sigma\rangle x$ well-formed. By ind. hyp. and Lemma $2.5, \vdash\left\langle\Sigma_{1}\right.$, let $x$ be [] in $\left.\Sigma_{2}[x]\right\rangle N \Downarrow\left\langle\Sigma_{3}\right.$, let $x$ be [] in $\left.\Sigma_{2}[x], \Theta\right\rangle V$ with $\left\lceil\Sigma_{3}\right.$, let $x$ be [] in $\left.\Sigma_{2}[x], \Theta\right\rceil=\Psi^{\prime}$. Thus we deduce $\vdash\langle\Sigma\rangle x \Downarrow\left\langle\Sigma_{3}, \Theta\right.$, let $x$ be $V$ in []$\left., \Sigma_{2}\right\rangle V$.

We prove the reduction semantics correct with respect to the natural semantics without going through the instrumented natural semantics. We first prove three useful lemmas. Lemma 2.6 proves that irrelevant evaluation contexts are replaceable. It lets us prove Lemmas 2.7 and 2.8. The former proves that reductions at the function position inside application can be recast outside the application. The latter proves that local reductions inside a let-binding can be recast as top-level reductions. We use the notation $M \rightarrow^{n} N$ to denote that $M$ reduces into $N$ in $n$ steps.

\section{Lemma 2.6}

For any $\Theta, E$ and $x$ such that $\Theta[E[x]]$ is a program and $x$ is not in $L B V(E)$, if $\Theta[E[x]] \rightarrow^{n} \Theta^{\prime}[E[V]]$, then for any $E^{\prime}$ such that $\Theta\left[E^{\prime}[x]\right]$ is a program and $x$ is not in $L B V\left(E^{\prime}\right), \Theta\left[E^{\prime}[x]\right] \rightarrow^{n} \Theta^{\prime}\left[E^{\prime}[V]\right]$.

\section{Proof}

By induction on $n$. Let $\Theta=\Theta_{1}$, let $x$ be $M$ in [], $\Theta_{2}$ with $x$ not in $L B V\left(\Theta_{2}\right)$. We perform case analysis on the possible reductions of $M$.

- The case in which $M$ is an answer is easy.

- The case in which $M$ (one-step) reduces independent of the context is immediate by induction.

- Suppose $M=E_{1}\left[x_{1}\right]$ and $x_{1}$ is not in $\operatorname{LBV}\left(E_{1}\right)$ and we have

$\Theta_{1}\left[\right.$ let $x$ be $E_{1}\left[x_{1}\right]$ in $\left.\Theta_{2}[E[x]]\right] \rightarrow^{n_{1}} \Theta_{1}^{\prime}\left[\right.$ let $x$ be $E_{1}\left[V_{1}\right]$ in $\left.\Theta_{2}[E[x]]\right] \rightarrow^{n_{2}}$ $\Theta^{\prime}[E[V]]$

Then by ind. hyp., we have

$\Theta_{1}\left[\right.$ let $x$ be $E_{1}\left[x_{1}\right]$ in $\left.\Theta_{2}\left[E^{\prime}[x]\right]\right] \rightarrow^{n_{1}} \Theta_{1}^{\prime}\left[\right.$ let $x$ be $E_{1}\left[V_{1}\right]$ in $\left.\Theta_{2}\left[E^{\prime}[x]\right]\right] \rightarrow^{n_{2}}$ $\Theta^{\prime}\left[E^{\prime}[V]\right]$

We introduce a notion of rooted reductions to identify a particular intermediate step in reductions: a reduction $M \rightarrow M^{\prime}$ is $\beta_{\text {need }}$-rooted with argument $N$ if $M=$ $\Theta\left[\left(\lambda x . N^{\prime}\right) N\right]$ and $M^{\prime}=\Theta\left[\right.$ let $x$ be $N$ in $\left.N^{\prime}\right]$. A reduction sequence $M \rightarrow M^{\prime}$ preserves a $\beta_{\text {need }}$-root with argument $N$ if none of (one-step) reductions in the sequence is $\beta_{\text {need }}$ rooted with argument $N$. Intuitively, if $\Theta[M N] \rightarrow M^{\prime}$ preserves a $\beta_{\text {need }}$-root with argument $N$, then all the reductions only occur at $M$ or in the environment $\Theta$.

\section{Lemma 2.7}

For any $\Theta, M$ and $N$ such that $\Theta[M N]$ is a program, if $\Theta[M N] \rightarrow^{n} \Theta^{\prime}[V N]$ and the reduction sequence preserves a $\beta_{\text {need }}$-root with argument $N$, then $\Theta[M] \rightarrow^{n^{\prime}} \Theta^{\prime}[V]$ with $n^{\prime} \leqslant n$. 
Proof

By induction on $n$ with case analysis on the possible reductions of $M$.

- The case in which $M$ is an answer is easy.

- The case in which $M$ reduces independent of the context is immediate by induction.

- Suppose $M=E[x]$ and $x$ is not in $L B V(E)$ and we have

$$
\Theta[(E[x]) N] \rightarrow^{n_{1}} \Theta_{1}[(E[V]) N] \rightarrow^{n_{2}} \Theta^{\prime}[V N]
$$

Then by Lemma 2.6 followed by ind. hyp., we have

$$
\Theta[E[x]] \rightarrow^{n_{1}} \Theta_{1}[E[V]] \rightarrow^{n_{2}^{\prime}} \Theta^{\prime}[V], \text { where } n_{2}^{\prime} \leqslant n_{2} .
$$

\section{Lemma 2.8}

For any $\Theta, x, M$ and $E$ such that $\Theta$ [let $x$ be $M$ in $E[x]]$ is a program and $x$ is not in $L B V(E)$, if $\Theta[$ let $x$ be $M$ in $E[x]] \rightarrow^{n} \Theta^{\prime}[$ let $x$ be $V$ in $E[x]]$, then $\Theta[M] \rightarrow^{n^{\prime}} \Theta^{\prime}[V]$ with $n^{\prime} \leqslant n$.

Proof

By induction on $n$ with case analysis on the possible reductions of $M$.

- The case in which $M$ is an answer is easy.

- The case in which $M$ reduces independent of the context is immediate by induction.

- Suppose $M=E^{\prime}\left[x^{\prime}\right]$ and $x^{\prime}$ is not in $L B V\left(E^{\prime}\right)$ and we have

$\Theta\left[\right.$ let $x$ be $E^{\prime}\left[x^{\prime}\right]$ in $\left.E[x]\right] \rightarrow^{n_{1}} \Theta_{1}\left[\right.$ let $x$ be $E^{\prime}\left[V^{\prime}\right]$ in $\left.E[x]\right] \rightarrow^{n_{2}} \Theta^{\prime}[$ let $x$ be $V$ in $E[x]]$

Then by Lemma 2.6 followed by ind. hyp., we have

$$
\Theta\left[E^{\prime}\left[x^{\prime}\right]\right] \rightarrow^{n_{1}} \Theta_{1}\left[E^{\prime}\left[V^{\prime}\right]\right] \rightarrow^{n_{2}^{\prime}} \Theta^{\prime}[V] \text { where } n_{2}^{\prime} \leqslant n_{2} .
$$

Now we are ready to prove the reduction semantics correct with respect to the natural semantics, using the above three lemmas to have induction go through.

\section{Proposition 2.3}

For any program $M$, if $M \rightarrow A$, then for any $X$, there exist $\Theta$ and $V$ such that $\Theta[V]$ and $A$ belong to the same $\alpha$-equivalence class and \langle\rangle $M \Downarrow_{X}\langle\lceil\Theta\rceil\rangle V$.

\section{Proof}

Without loss of generality, we assume $\Theta[V]$ and $A$ are syntactically identical. We prove by induction on the length of the reductions of $M$. Let $M=\Theta^{\prime}\left[M^{\prime}\right]$ with $M^{\prime} \neq$ let $x$ be $N^{\prime}$ in $N$. We perform case analysis on $M^{\prime}$.

- The case of abstraction is obvious.

- The case of application. Suppose $M^{\prime}=M_{1} M_{2}$ and we have

$$
\Theta^{\prime}\left[M_{1} M_{2}\right] \rightarrow \Theta_{1}\left[\left(\lambda x . M_{3}\right) M_{2}\right] \rightarrow \Theta_{1}\left[\text { let } x \text { be } M_{2} \text { in } M_{3}\right] \rightarrow \Theta[V]
$$

By Lemma 2.7 and ind. hyp., \langle\rangle$\Theta^{\prime}\left[M_{1}\right] \Downarrow_{X}\left\langle\left\lceil\Theta_{1}\right\rceil\right\rangle \lambda x . M_{3}$. By ind. hyp, \langle\rangle$\Theta_{1}$ [let $x$ be $M_{2}$ in $\left.M_{3}\right] \Downarrow_{X}\langle[\Theta\rceil\rangle V$. Thus we deduce \langle\rangle$\Theta^{\prime}\left[M_{1} M_{2}\right] \Downarrow_{X}\langle[\Theta\rceil\rangle V$.

- The case of a variable. Suppose $M^{\prime}=x$ and $\Theta^{\prime}=\Theta_{1}$, let $x$ be $N$ in [], $\Theta_{2}$ and we have

$\Theta_{1}\left[\right.$ let $x$ be $N$ in $\left.\Theta_{2}[x]\right] \rightarrow \Theta_{1}^{\prime}$ [let $x$ be $V$ in $\left.\Theta_{2}[x]\right] \rightarrow \Theta_{1}^{\prime}$ [let $x$ be $V$ in $\left.\Theta_{2}[V]\right]$ 


$\begin{array}{llll}\text { Expressions } & M, N & := & x|\lambda x \cdot M| M N \mid \text { let } \operatorname{rec} D \text { in } M \mid \bullet \\ \text { Definitions } & D & := & \varepsilon \mid D, x \text { be } M \\ \text { Values } & V & := & \lambda x . M \mid \bullet \\ \text { Answers } & A & := & V \mid \text { let rec } D \text { in } A \\ \text { Contexts } & E & := & {[|E M| \text { let rec } D \text { in } E} \\ & & \mid & \text { let rec } x \text { be } E, D \text { in } E^{\prime}[x] \\ & & \mid & \text { let rec } x^{\prime} \text { be } E, D\left[x, x^{\prime}\right], D \text { in } E^{\prime}[x] \\ \text { Dependencies } & D\left[x, x^{\prime}\right] & := & x \text { be } E\left[x^{\prime}\right] \\ & & \mid & D\left[x, x^{\prime \prime}\right], x^{\prime \prime} \text { be } E\left[x^{\prime}\right]\end{array}$

Fig. 9. Syntax of $\lambda_{\text {letrec }}$.

$\beta_{\text {need }}: \quad(\lambda x . M) N \underset{\text { need }}{\longrightarrow}$ let rec $x$ be $N$ in $M$

lift: $\quad($ let $\operatorname{rec} D$ in $A) N \underset{\text { need }}{\longrightarrow}$ let rec $D$ in $A N$

deref: $\quad$ let rec $x$ be $V, D$ in $E[x] \underset{\text { need }}{\longrightarrow}$ let rec $x$ be $V, D$ in $E[V]$

deref $_{\text {env }}$ : let rec $D\left[x, x^{\prime}\right], x^{\prime}$ be $V, D$ in $E[x] \underset{\text { need }}{\longrightarrow}$ let rec $D[x, V], x^{\prime}$ be $V, D$ in $E[x]$

assoc: let rec $x$ be (let rec $D$ in $A$ ), $D^{\prime}$ in $E[x] \underset{\text { need }}{\longrightarrow}$ let rec $D, x$ be $A, D^{\prime}$ in $E[x]$

assocenv $_{\text {en }}$ let rec $x^{\prime}$ be (let rec $D$ in $\left.A\right), D\left[x, x^{\prime}\right], D^{\prime}$ in $E[x] \underset{\text { need }}{\longrightarrow}$ let rec $D, x^{\prime}$ be $A, D\left[x, x^{\prime}\right], D^{\prime}$ in $E[x]$

error: $\quad$ let rec $D[x, x], D$ in $E[x] \underset{\text { need }}{\longrightarrow}$ let rec $D[x, \bullet], D$ in $E[x]$

error $_{\text {env }}$ : let rec $D\left[x^{\prime}, x^{\prime}\right], D^{\prime}\left[x, x^{\prime}\right], D$ in $E[x] \underset{\text { need }}{\longrightarrow}$ let rec $D\left[x^{\prime}, \bullet\right], D^{\prime}\left[x, x^{\prime}\right], D$ in $E[x]$

error $_{\beta}: \bullet M \underset{\text { need }}{\longrightarrow} \bullet$

Fig. 10. Reduction semantics for $\lambda_{\text {letrec}}$.

By Lemma 2.8 and ind. hyp., \langle\rangle$\Theta_{1}[N] \Downarrow_{X \cup\{x\} \cup \operatorname{dom}\left(\left[\Theta_{2}\right\rceil\right)}\left\langle\left\lceil\Theta_{1}^{\prime}\right\rceil\right\rangle V$, from which we deduce \langle\rangle$\Theta^{\prime}[x] \Downarrow_{X}\left\langle\left\lceil\Theta_{1}^{\prime}\right.\right.$, let $x$ be $V$ in []$\left.\left., \Theta_{2}\right\rceil\right\rangle V$.

Collecting all propositions together, we prove the equivalence of the two semantics.

Theorem 2.1

For any program $M$, the following two conditions hold:

(1) if $M \rightarrow A$, then there exist $\Theta$ and $V$ such that $\Theta[V]$ and $A$ belong to the same $\alpha$-equivalence class and \langle\rangle $M \Downarrow_{\emptyset}\langle\lceil\Theta\rceil\rangle V$;

(2) if \langle\rangle $M \Downarrow_{\emptyset}\langle\Psi\rangle V$, then $M \rightarrow \Theta[V]$, where $\lceil\Theta\rceil=\Psi$.

\section{Proof}

(1) By Proposition 2.3.

(2) By Proposition 2.2 and Lemma 2.5, $\vdash\langle\rangle M \Downarrow\langle\Theta\rangle V$ with $\lceil\Theta\rceil=\Psi$. By Proposition 2.1, $M \rightarrow \Theta[V]$.

\section{Call-by-need letrec calculus $\lambda_{\text {letrec }}$}

In this section we extend the equivalence result to the cyclic (recursive) calculus.

\subsection{Syntax and semantics}

The syntax of the call-by-need letrec calculus $\lambda_{\text {letrec }}$ is defined in Figure 9. The reduction and natural semantics are defined in Figures 10 and 11 respectively. No 


$$
\begin{gathered}
\text { Value } \\
\langle\Psi\rangle V \Downarrow\langle\Psi\rangle V \\
\text { Application } \\
\frac{\langle\Psi\rangle M_{1} \Downarrow\langle\Phi\rangle \lambda x . N \quad\left[\Phi\left[x^{\prime} \mapsto M_{2}\right]\right\rangle N\left[x^{\prime} / x\right] \Downarrow\left\langle\Psi^{\prime}\right\rangle V \quad x^{\prime} \text { fresh }}{\langle\Psi\rangle M_{1} M_{2} \Downarrow\left\langle\Psi^{\prime}\right\rangle V} \\
\text { Variable } \\
\frac{\langle\Psi[x \mapsto \bullet]\rangle \Psi(x) \Downarrow\langle\Phi\rangle V}{\langle\Psi\rangle x \Downarrow\langle\Phi[x \mapsto V]\rangle V} \\
\text { Letrec } \\
\frac{\left\langle\Psi\left[x_{i}^{\prime} \mapsto M_{i}^{\prime}\right]_{i \in\{1, \ldots, n\}}\right\rangle N^{\prime} \Downarrow\langle\Phi\rangle V \quad x_{1}^{\prime}, \ldots, x_{n}^{\prime} \text { fresh }}{\langle\Psi\rangle \text { let rec } x_{1} \text { be } M_{1}, \ldots, x_{n} \text { be } M_{n} \text { in } N \Downarrow\langle\Phi\rangle V} \\
\text { Error } \\
\frac{\langle\Psi\rangle M_{1} \Downarrow\langle\Phi\rangle \bullet}{\langle\Psi\rangle M_{1} M_{2} \Downarrow\langle\Phi\rangle \bullet}
\end{gathered}
$$

Fig. 11. Natural semantics for $\lambda_{\text {letrec }}$.

ordering among bindings in $D$ is assumed. Metavariables $\Psi$ and $\Phi$ range over finite mappings from variables to expressions. Here we do not assume any ordering among bindings in heaps. In particular, a heap may contain cyclic structure such as $\left\langle x_{1} \mapsto \lambda y . x_{2} y, x_{2} \mapsto \lambda y \cdot x_{1} y\right\rangle$ and $\langle x \mapsto y, y \mapsto x\rangle$. In the natural semantics, the notation $\Psi\left[x_{i} \mapsto M_{i}\right]_{i \in\{1, \ldots, n\}}$ denotes mapping extension. Precisely,

$$
\Psi\left[x_{i} \mapsto M_{i}\right]_{i \in\{1, \ldots, n\}}(x)= \begin{cases}M_{i} & \text { when } x=x_{i} \text { for some } i \text { in } 1, \ldots, n, \\ \Psi(x) & \text { otherwise. }\end{cases}
$$

We write $\Psi[x \mapsto M]$ to denote a single extension of $\Psi$ with $M$ at $x$. In rule Letrec of Figure $11, M_{i}^{\prime}$ 's and $N^{\prime}$ denote expressions obtained from $M_{i}$ 's and $N$, respectively, by substituting $x_{i}^{\prime}$ 's for $x_{i}^{\prime}$ 's. We may abbreviate $\langle\Psi\rangle M$, where $\Psi$ is an empty mapping, i.e. the domain of $\Psi$ is empty, to \langle\rangle $M$. We adapt the definition of free variables in Figure 4 for $\lambda_{\text {letrec }}$ by replacing the rule for let with the following rule:

$$
\begin{aligned}
& F V\left(\text { let rec } x_{1} \text { be } M_{1}, \ldots, x_{n} \text { be } M_{n} \text { in } N\right) \\
& \quad=\left(F V\left(M_{1}\right) \cup \ldots \cup F V\left(M_{n}\right) \cup F V(N)\right) \backslash\left\{x_{1}, \ldots, x_{n}\right\}
\end{aligned}
$$

The reduction semantics is mostly identical to the previous presentation by Ariola and Felleisen (1997), except that we elaborately deal with 'undefinedness', which arises due to direct cycles such as let rec $x$ be $x$ in $M$. Undefinedness represents provable divergences. In our reduction semantics undefinedness, or black holes • are produced and propagated explicitly, in a spirit similar to Wright and Felleisen's treatment of exceptions in a reduction calculus (Wright \& Felleisen 1994). Rules error and error env produce black holes. Applying a black hole to an expression results in a black hole $\left(\right.$ error $\left._{\beta}\right)$. A value may be an abstraction or a black hole. Thus rules lift, deref, deref env, assoc and $\operatorname{assoc}_{\text {env }}$ can be exercised to propagate black holes. Explicit handling of black holes facilitates inductive reasoning. Again the reduction semantics works with $\alpha$-equivalence classes of expressions. The following lemma states the unique-decomposition property for $\lambda_{\text {letrec }}$ and is proved by induction on $M$. 
let rec $x$ be $f x, f$ be $\lambda y . y$ in $x$

$\rightarrow$ let rec $x$ be $(\lambda y \cdot y) x, f$ be $\lambda y . y$ in $x$

$\rightarrow$ let rec $x$ be (let rec $y$ be $x$ in $y$ ), $f$ be $\lambda y \cdot y$ in $x$

$\rightarrow$ let rec $x$ be (let rec $y$ be $\bullet$ in $y$ ), $f$ be $\lambda y \cdot y$ in $x$

$\rightarrow$ let rec $x$ be (let rec $y$ be $\bullet$ in $\bullet$ ), $f$ be $\lambda y . y$ in $x$

$\rightarrow$ let rec $y$ be $\bullet, x$ be $\bullet, f$ be $\lambda y . y$ in $x$

$\rightarrow$ let rec $y$ be $\bullet, x$ be $\bullet, f$ be $\lambda y . y$ in $\bullet$

Fig. 12. The reduction sequence for let rec $x$ be $f x, f$ be $\lambda y . y$ in $x$.

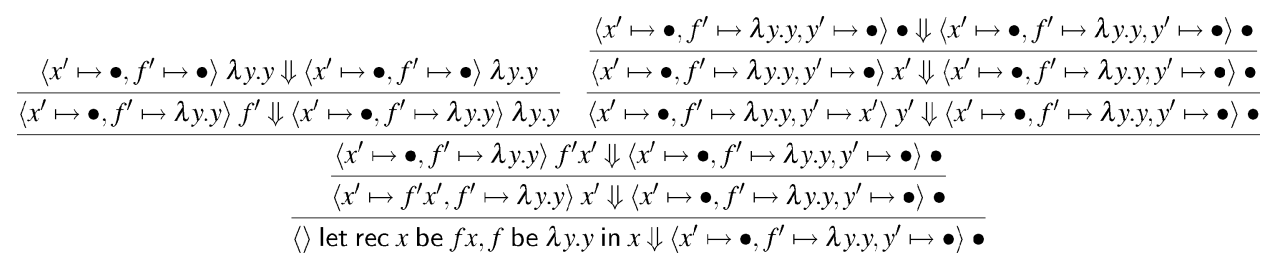

Fig. 13. The derivation for let rec $x$ be $f x, f$ be $\lambda y . y$ in $x$.

\section{Lemma 3.1}

For any program $M$, either $M$ is an answer or there exist a unique context $E$ and redex $N$ such that $M=E[N]$.

The natural semantics is very much inspired by that of Sestoft (1997) and hence by that of Launchbury (1993). We revise Sestoft's semantics in the following two points to draw a direct connection with the reduction semantics. Firstly, in accordance with the reduction semantics, our natural semantics may return black holes. In Variable rule, $x$ is bound to $\bullet$ while the bound expression to $x$ is evaluated. For instance, \langle\rangle let rec $x$ be $x$ in $x \Downarrow\left\langle x^{\prime} \mapsto \bullet\right\rangle \bullet$ is deduced in our formulation. Sestoft's formulation removes the binding of $x$ from the heap during its evaluation; thus evaluation involving direct cycles 'gets stuck'; i.e. no derivation is possible when direct cycles are encountered. Since we do not remove bindings from heaps, freshness conditions are locally checkable without extra variable tracking. Secondly, we do not precompile expressions into 'normalized' ones. Our semantics works with full lambda expressions with letrec, where function arguments may be any expression, not only variables.

The notation $\operatorname{dom}(\Psi)$ denotes the domain of $\Psi$. A configuration $\langle\Psi\rangle M$ is closed if $F V(M) \subseteq \operatorname{dom}(\Psi)$, and for any $x$ in $\operatorname{dom}(\Psi), F V(\Psi(x)) \subseteq \operatorname{dom}(\Psi)$.

Example Figures 12 and 13 present the reduction sequence and the derivation for the expression let rec $x$ be $f x, f$ be $\lambda y . y$ in $x$ respectively. We deliberately chose a black hole producing expression to demonstrate the difference of our formulation from those of Ariola and Felleisen (1997) and Sestoft (1997).

\subsection{Equivalence of the two semantics}

We prove equivalence of the two semantics for $\lambda_{\text {letrec }}$ in similar steps as those for $\lambda_{\text {let }}$ and use an instrumented natural semantics defined in Figure 14. The notation 


$$
\begin{aligned}
& \text { Frames } \quad F \quad::=[] M \mid \text { let rec } D \text { in [] | let rec } D_{x}, D \text { in } E[x] \\
& \text { Structured heaps } \quad \Sigma \quad::=\varepsilon \mid \Sigma, F \\
& \begin{array}{llll} 
& D_{x} & ::= & x \text { be }[] \mid D\left[x, x^{\prime}\right], x^{\prime} \text { be } \\
\text { Letrec }^{\prime} s & \Theta & ::= & \varepsilon \mid \Theta, \text { let rec } D \text { in }[]
\end{array} \\
& \text { Val } \\
& \vdash\langle\Sigma\rangle V \Downarrow\langle\Sigma\rangle V \\
& \text { App } \\
& \frac{\vdash\left\langle\Sigma,[] M_{2}\right\rangle M_{1} \Downarrow\left\langle\Sigma_{1},[] M_{2}, \Theta\right\rangle \lambda x . N \quad \vdash\left\langle\Sigma_{1}, \Theta \text {, let rec } x^{\prime} \text { be } M_{2} \text { in }[]\right\rangle N\left[x^{\prime} / x\right] \Downarrow\left\langle\Sigma_{2}\right\rangle V \quad x^{\prime} \text { fresh }}{\vdash\langle\Sigma\rangle M_{1} M_{2} \Downarrow\left\langle\Sigma_{2}\right\rangle V} \\
& \text { Letrecin } \\
& \frac{\vdash\left\langle\Sigma \text {, let rec } x_{1}^{\prime} \text { be } M_{1}^{\prime}, \ldots, x_{n}^{\prime} \text { be } M_{n}^{\prime} \text { in }[]\right\rangle N^{\prime} \Downarrow\left\langle\Sigma^{\prime}\right\rangle V \quad x_{1}^{\prime}, \ldots, x_{n}^{\prime} \text { fresh }}{\vdash\langle\Sigma\rangle \text { let rec } x_{1} \text { be } M_{1}, \ldots, x_{n} \text { be } M_{n} \text { in } N \Downarrow\left\langle\Sigma^{\prime}\right\rangle V} \\
& \text { Var } \\
& \frac{\vdash\left\langle\Sigma \text {, let rec } x \text { be }[], D \text { in } \Sigma_{1}[x]\right\rangle M \Downarrow\left\langle\Sigma^{\prime} \text {, let rec } x \text { be [], } D^{\prime} \text { in } \Sigma_{1}[x], \Theta\right\rangle V}{\vdash\left\langle\Sigma \text {, let rec } x \text { be } M, D \text { in [], } \Sigma_{1}\right\rangle x \Downarrow\left\langle\Sigma^{\prime} \text {, let rec } \bar{\Theta}, x \text { be } V, D^{\prime} \text { in [], } \Sigma_{1}\right\rangle V} \\
& \operatorname{Var}_{\text {env }} \\
& \frac{\vdash\left\langle\Sigma \text {, let rec } x \text { be }[], D_{x^{\prime}}\left[\Sigma_{1}[x]\right], D \text { in } E\left[x^{\prime}\right]\right\rangle M \Downarrow\left\langle\Sigma^{\prime} \text {, let rec } x \text { be }[], D_{x^{\prime}}\left[\Sigma_{1}[x]\right], D^{\prime} \text { in } E\left[x^{\prime}\right], \Theta\right\rangle V}{\vdash\left\langle\Sigma \text {, let rec } x \text { be } M, D_{x^{\prime}}, D \text { in } E\left[x^{\prime}\right], \Sigma_{1}\right\rangle x \Downarrow\left\langle\Sigma^{\prime} \text {, let rec } \bar{\Theta}, x \text { be } V, D_{x^{\prime}}, D^{\prime} \text { in } E\left[x^{\prime}\right], \Sigma_{1}\right\rangle V} \\
& \frac{\begin{array}{c}
E r r_{\text {var }} \\
x \in D_{x^{\prime}}
\end{array}}{\vdash\left\langle\Sigma \text {, let rec } D, D_{x^{\prime}} \text { in } E\left[x^{\prime}\right], \Sigma^{\prime}\right\rangle x \Downarrow\left\langle\Sigma \text {, let rec } D, D_{x^{\prime}} \text { in } E\left[x^{\prime}\right], \Sigma^{\prime}\right\rangle \bullet} \\
& \operatorname{Err}_{\beta} \\
& \frac{\vdash\left\langle\Sigma,[] M_{2}\right\rangle M_{1} \Downarrow\left\langle\Sigma^{\prime},[] M_{2}, \Theta\right\rangle \bullet}{\vdash\langle\Sigma\rangle M_{1} M_{2} \Downarrow\left\langle\Sigma^{\prime}, \Theta\right\rangle \bullet}
\end{aligned}
$$

Fig. 14. Instrumented natural semantics for $\lambda_{\text {letrec }}$.

$\bar{\Theta}$ denotes the flattening of $\Theta$. Or

$$
\bar{\epsilon}=\epsilon \quad \bar{\Theta} \text {, let rec } D \text { in }[]=\bar{\Theta}, D
$$

The notation $x \in D_{x^{\prime}}$ denotes that $x$ is letrec-bound in $D_{x^{\prime}}$; i.e. either $x$ be [] or $x$ be $M$ is in $D_{x^{\prime}}$. In rule Letrecin, $M_{i}^{\prime}$ s s and $N^{\prime}$ denote expressions obtained from $M_{i}$ 's and $N$ by substituting $x_{i}^{\prime}$ 's for $x_{i}^{\prime}$ 's, respectively.

Here a frame may be let rec $D$ in [] or let rec $D_{x}, D$ in $E[x]$, instead of let $x$ be $M$ in [] or let $x$ be [] in $E[x]$. We need to adjust the definitions of well-formedness for structured heaps and structured configurations. The notation $L B V(\Sigma)$ denotes the set of variables letrec-bound in frames of $\Sigma$. Or

$$
\begin{aligned}
L B V(\epsilon) & =\emptyset \\
L B V(\Sigma,[] M) & =L B V(\Sigma) \\
L B V(\Sigma, \text { let rec } D \text { in }[]) & =L B V(\Sigma) \cup L B V(D) \\
L B V\left(\Sigma, \text { let rec } D, D_{x} \text { in } M\right) & =L B V(\Sigma) \cup L B V\left(D, D_{x}\right) \\
L B V(D, x \text { be } M) & =L B V(D) \cup\{x\} \\
L B V(D, x \text { be }[]) & =L B V(D) \cup\{x\}
\end{aligned}
$$


The notations $\operatorname{Exp}(F)$ and $\operatorname{Exp}(\Sigma)$ respectively denote the sets of expressions that $F$ and $\Sigma$ contain. Or

$$
\begin{aligned}
\operatorname{Exp}([] M) & =\{M\} \\
\operatorname{Exp}(\text { let rec } D \text { in }[]) & =\operatorname{Exp}(D) \\
\operatorname{Exp}\left(\text { let rec } D, D_{x} \text { in } M\right) & =\{M\} \cup \operatorname{Exp}\left(D, D_{x}\right) \\
\operatorname{Exp}(\epsilon) & =\emptyset \\
\operatorname{Exp}(D, x \text { be } M) & =\operatorname{Exp}(D) \cup\{M\} \\
\operatorname{Exp}(D, x \text { be }[]) & =\operatorname{Exp}(D) \\
\operatorname{Exp}(\Sigma, F) & =\operatorname{Exp}(\Sigma) \cup \operatorname{Exp}(F)
\end{aligned}
$$

A structured heap $\Sigma$ is well-formed if it is an empty sequence, or else $\Sigma=\Sigma^{\prime}, F$, and $\Sigma^{\prime}$ is well-formed and one of the following conditions hold:

(1) $F=[] M$ and $F V(M) \subseteq L B V(\Sigma)$;

(2) $F=$ let rec $x_{1}$ be $M_{1}, \ldots, x_{n}$ be $M_{n}$ in [] and $F V\left(M_{i}\right) \subseteq L B V(\Sigma)$ for all $i$ 's, and $x_{1}, \ldots, x_{n}$ are pairwise distinctly named, and all $x_{i}$ 's are distinct from any of $\operatorname{LBV}\left(\Sigma^{\prime}\right)$;

(3) $F=$ let rec $x$ be [], $x_{1}$ be $M_{1}, \ldots, x_{n}$ be $M_{n}$ in $N$ and $F V(N) \subseteq L B V(\Sigma)$ and $F V\left(M_{i}\right) \subseteq L B V(\Sigma)$ for all $i$ 's, and $x, x_{1}, \ldots, x_{n}$ are pairwise distinctly named, and all $x_{i}$ 's and $x$ are distinct from any of $L B V\left(\Sigma^{\prime}\right)$.

A structured configuration $\langle\Sigma\rangle M$ is well-formed if $\Sigma$ is well-formed and $F V(M) \subseteq$ $\operatorname{LBV}(\Sigma)$.

We use the same definition as in the previous section for the translation $\lfloor\cdot\rfloor$ from structured heaps to contexts:

$$
\lfloor\epsilon\rfloor=[] \quad\lfloor\Sigma, F\rfloor=\lfloor\Sigma\rfloor[F]
$$

Again we may identify $\Sigma$ with $\lfloor\Sigma\rfloor$ and thus write $\Sigma[M]$ to denote $\lfloor\Sigma\rfloor[M]$. The following lemma is proved by induction on the structure of $\Sigma$.

\section{Lemma 3.2}

For any well-formed configuration $\langle\Sigma\rangle M, \Sigma[M]$ is a program.

Let's look at the inference rules in Figure 14. The first four rules are equivalent to the previous four rules in Figure 7. Whereas Var corresponds to the production let rec $x$ be $E, D$ in $E^{\prime}[x]$ of evaluation contexts, $\operatorname{Var}_{\text {env }}$ corresponds to the production let rec $x^{\prime}$ be $E, D\left[x, x^{\prime}\right], D$ in $E^{\prime}[x]$. Err var mediates between the natural and the reduction semantics when a black hole is produced. Indeed variables letrecbound in $D_{x}$ correspond to variables bound to $\bullet$ in a heap in the natural semantics. The instrumented natural semantics keeps the original expressions bound to the variables to facilitate reconstructing reduction sequences from its derivations. $\operatorname{Err}_{\beta}$ is almost the same as the original rule Error $_{\beta}$ in Figure 11.

\section{Lemma 3.3}

If $\langle\Sigma\rangle M$ is well-formed and $\vdash\langle\Sigma\rangle M \Downarrow\left\langle\Sigma^{\prime}\right\rangle V$, then $\left\langle\Sigma^{\prime}\right\rangle V$ is well-formed.

\section{Proof}

By induction on the derivation of $\langle\Sigma\rangle M \Downarrow\left\langle\Sigma^{\prime}\right\rangle V$. 
Easy induction proves the instrumented natural semantics correct with respect to the reduction semantics.

Proposition 3.1

If $\langle\Sigma\rangle M$ is well-formed and $\vdash\langle\Sigma\rangle M \Downarrow\left\langle\Sigma^{\prime}\right\rangle V$, then $\Sigma[M] \rightarrow \Sigma^{\prime}[V]$.

\section{Proof}

By induction on the derivation of $\vdash\langle\Sigma\rangle M \Downarrow\left\langle\Sigma^{\prime}\right\rangle V$ with case analysis on the last rule used.

- The case of $V a l$ is obvious.

- The case of App. Suppose we deduce $\vdash\langle\Sigma\rangle M_{1} M_{2} \Downarrow\left\langle\Sigma^{\prime}\right\rangle V$ from $\vdash\left\langle\Sigma\right.$, [] $\left.M_{2}\right\rangle$ $M_{1} \Downarrow\left\langle\Sigma_{1},[] M_{2}, \Theta\right\rangle \lambda x . N$ and $\vdash\left\langle\Sigma_{1}, \Theta\right.$, let rec $x^{\prime}$ be $M_{2}$ in [] $\rangle\left[x^{\prime} / x\right] \Downarrow\left\langle\Sigma^{\prime}\right\rangle V$. Then we have

$\Sigma\left[M_{1} M_{2}\right]$

$\rightarrow \Sigma_{1}\left[(\Theta[\lambda x . N]) M_{2}\right]$ by ind. hyp.

$\rightarrow \Sigma_{1}\left[\Theta\left[(\lambda x . N) M_{2}\right]\right]$ by lift

$\rightarrow \Sigma_{1}\left[\Theta\left[\right.\right.$ let rec $x^{\prime}$ be $M_{2}$ in $\left.\left.N\left[x^{\prime} / x\right]\right]\right]$ by $\beta_{\text {need }}$

$\rightarrow \Sigma^{\prime}[V]$ by ind. hyp.

- The case of Letrecin is immediate by induction.

- The case of Var. Suppose we deduce $\vdash\left\langle\Sigma\right.$, let rec $x$ be $M, D$ in [], $\left.\Sigma_{1}\right\rangle x \Downarrow$ $\left\langle\Sigma_{2}\right.$, let rec $\bar{\Theta}, x$ be $V, D^{\prime}$ in [], $\left.\Sigma_{1}\right\rangle V$ from $\vdash\left\langle\Sigma\right.$, let rec $x$ be [], $D$ in $\left.\Sigma_{1}[x]\right\rangle M \Downarrow$ $\left\langle\Sigma_{2}\right.$, let rec $x$ be [], $D^{\prime}$ in $\left.\Sigma_{1}[x], \Theta\right\rangle V$. Then we have

$\Sigma\left[\right.$ let rec $x$ be $M, D$ in $\left.\Sigma_{1}[x]\right]$

$\rightarrow \Sigma_{2}\left[\right.$ let rec $x$ be $\Theta[V], D^{\prime}$ in $\left.\Sigma_{1}[x]\right]$ by ind. hyp.

$\rightarrow \Sigma_{2}\left[\right.$ let rec $\bar{\Theta}, x$ be $V, D^{\prime}$ in $\left.\Sigma_{1}[x]\right]$ by assoc

$\rightarrow \Sigma_{2}\left[\right.$ let rec $\bar{\Theta}, x$ be $V, D^{\prime}$ in $\left.\Sigma_{1}[V]\right]$ by deref

- The case of $V a r_{\text {env }}$ is similar to the above Var case, where we use $a s s o c_{e n v}$ and deref $_{\text {env }}$ instead of assoc and deref, respectively.

- The case of $E r r_{v a r}(1)$. Suppose $x=x^{\prime}$ and we deduce $\vdash\left\langle\Sigma\right.$,let rec $D, D_{x}$ in $E[x]$, $\left.\Sigma^{\prime}\right\rangle x \Downarrow\left\langle\Sigma\right.$, let rec $D, D_{x}$ in $\left.E[x], \Sigma^{\prime}\right\rangle \bullet$. The side-condition $x \in D_{x}$ implies $D_{x}\left[\Sigma^{\prime}[x]\right]=D[x, x]$. Thus we have $\Sigma\left[\right.$ let rec $D, D_{x}\left[\Sigma^{\prime}[x]\right]$ in $\left.E[x]\right] \rightarrow$ $\Sigma\left[\right.$ let rec $D, D_{x}\left[\Sigma^{\prime}[\bullet]\right]$ in $\left.E[x]\right]$ by error.

- The case of $\operatorname{Err}_{v a r}$ (2). Suppose $x \neq x^{\prime}$ and we deduce $\vdash\left\langle\Sigma\right.$,let rec $D, D_{x^{\prime}}$ in $E\left[x^{\prime}\right]$, $\left.\Sigma^{\prime}\right\rangle x \Downarrow\left\langle\Sigma\right.$, let rec $D, D_{x^{\prime}}$ in $\left.E\left[x^{\prime}\right], \Sigma^{\prime}\right\rangle \bullet$. Then $x \in D_{x^{\prime}}$ implies $D_{x^{\prime}}\left[\Sigma^{\prime}[x]\right]=$ $D\left[x^{\prime}, x\right], D[x, x]$. Thus we have $\Sigma\left[\right.$ let $\operatorname{rec} D, D_{x^{\prime}}\left[\Sigma^{\prime}[x]\right]$ in $\left.E\left[x^{\prime}\right]\right] \rightarrow \Sigma\left[\right.$ let $\operatorname{rec} D, D_{x^{\prime}}$ $\left[\Sigma^{\prime}[\bullet]\right]$ in $\left.E\left[x^{\prime}\right]\right]$ by error ${ }_{\text {env }}$.

- The case of $\operatorname{Err}_{\beta}$ is easy and similar to App.

Next we prove the instrumented natural semantics correct with respect to the original natural semantics in Figure 11. Again this amounts to checking that in the instrumented natural semantics pushing and popping frames into heaps are properly balanced. The proof is similar to the previous one for Proposition 2.2, but we extend the preorder $\leqslant$ on structured heaps to take account of their cyclic structure.

To define the preorder $\leqslant$ on structured heaps, we use two auxiliary preorders. The preorder $\leqslant_{\mathscr{D}}$ on sequences of bindings is defined such that $D \leqslant \mathscr{D} D^{\prime}$ if $L B V(D) \subseteq$ $L B V\left(D^{\prime}\right)$. The preorder $\leqslant \mathscr{F}$ on frames is the smallest reflexive and transitive 
relation satisfying the condition that if $D \leqslant \mathscr{D} D^{\prime}$, then let rec $D_{x}, D$ in $E[x] \leqslant \mathscr{F}$ let rec $D_{x}, D^{\prime}$ in $E[x]$ and let rec $D$ in [] $\leqslant \mathscr{F}$ let rec $D^{\prime}$ in []. Then the preorder $\leqslant$ on structured heaps is defined such that $F_{1}, \ldots, F_{m} \leqslant F_{1}^{\prime}, \ldots, F_{n}^{\prime}$ if there is an injection $\imath$ from $\{1, \ldots, m\}$ to $\{1, \ldots, n\}$ satisfying the following three conditions:

(1) if $i<j$, then $\imath(i)<\imath(j)$;

(2) for all $i$ in $\{1, \ldots, m\}, F_{i} \leqslant \mathscr{F} F_{l(i)}^{\prime}$;

(3) for all $i$ in $\{1, \ldots, n\} \backslash \operatorname{ran}(\imath), F_{i}^{\prime}=$ let rec $D$ in [] for some $D$.

It is easy to check that $\leqslant$ is a preorder. The following lemma is proved by induction on the derivation of $\vdash\langle\Sigma\rangle M \Downarrow\left\langle\Sigma^{\prime}\right\rangle V$.

\section{Lemma 3.4}

If $\langle\Sigma\rangle M$ is well-formed and $\vdash\langle\Sigma\rangle M \Downarrow\left\langle\Sigma^{\prime}\right\rangle V$, then $\Sigma \leqslant \Sigma^{\prime}$.

We define translation $\lceil\cdot\rceil$ from structured heaps into sequences of bindings by

$$
\begin{aligned}
\lceil\epsilon\rceil & =\epsilon \\
\lceil\Sigma,[] M\rceil & =\lceil\Sigma\rceil \\
\lceil\Sigma, \text { let rec } D \text { in }[]\rceil & =\lceil\Sigma\rceil, D \\
\left\lceil\Sigma, \text { let rec } D, D_{x} \text { in } M\right\rceil & =\lceil\Sigma\rceil, D, x_{1} \text { be } \bullet, \ldots, x_{n} \text { be } \bullet
\end{aligned}
$$

where $\operatorname{LBV}\left(D_{x}\right)=\left\{x_{1}, \ldots, x_{n}\right\}$. We identify a sequence of bindings $D$ with a heap $\Psi$ such that $L B V(D)=\operatorname{dom}(\Psi)$, and for all $x$ in $\operatorname{dom}(\Psi), \Psi(x)=M$ iff $D$ contains $x$ be $M$. Thus $\lceil\Sigma\rceil$ denotes a heap.

We prove one basic result about the natural semantics: Lemma 3.5 states that extending heaps with irrelevant bindings does not affect derivations and is proved by routine induction. For mappings $\Psi, \Phi$ such that $\operatorname{dom}(\Psi)$ and $\operatorname{dom}(\Phi)$ are disjoint, the notation $\Psi \cup \Phi$ denotes their union, namely $\operatorname{dom}(\Psi \cup \Phi)=\operatorname{dom}(\Psi) \cup \operatorname{dom}(\Phi)$ and

$$
(\Psi \cup \Phi)(x)= \begin{cases}\Psi(x) & \text { when } x \in \operatorname{dom}(\Psi), \\ \Phi(x) & \text { when } x \in \operatorname{dom}(\Phi)\end{cases}
$$

\section{Lemma 3.5}

For any $\Psi, \Psi^{\prime}, \Phi$ and $M$ such that $\operatorname{dom}\left(\Psi^{\prime}\right)$ and $\operatorname{dom}(\Phi)$ are disjoint and $\langle\Psi\rangle M$ and $\left\langle\Psi \cup \Psi^{\prime}\right\rangle M$ are closed, $\langle\Psi\rangle M \Downarrow\langle\Phi\rangle V$ iff $\left\langle\Psi \cup \Psi^{\prime}\right\rangle M \Downarrow\left\langle\Phi \cup \Psi^{\prime}\right\rangle V$ and their derivations are of the same depth.

Proposition 3.2

If $\langle\Psi\rangle M$ is closed and $\langle\Psi\rangle M \Downarrow\langle\Phi\rangle V$, then for any $\Sigma$ such that $\lceil\Sigma\rceil=\Psi$ and $\langle\Sigma\rangle M$ is well-formed, $\vdash\langle\Sigma\rangle M \Downarrow\left\langle\Sigma^{\prime}\right\rangle V$ with $\left\lceil\Sigma^{\prime}\right\rceil=\Phi$.

\section{Proof}

By induction on the depth of the derivation of $\langle\Psi\rangle M \Downarrow\langle\Phi\rangle V$ with case analysis on the last rule used.

- The case of Value is obvious.

- The case of Application. Suppose $\lceil\Sigma\rceil=\Psi$ and $\langle\Sigma\rangle M_{1} M_{2}$ is well-formed and we deduce $\langle\Psi\rangle M_{1} M_{2} \Downarrow\left\langle\Psi^{\prime}\right\rangle V$ from $\langle\Psi\rangle M_{1} \Downarrow\langle\Phi\rangle \lambda x . N$ and $\left\langle\Phi\left[x^{\prime} \mapsto\right.\right.$ $\left.\left.M_{2}\right]\right\rangle N\left[x^{\prime} / x\right] \Downarrow\left\langle\Psi^{\prime}\right\rangle V$. By ind. hyp. and Lemma 3.4, $\vdash\left\langle\Sigma,[] M_{2}\right\rangle M_{1} \Downarrow$ 
$\left\langle\Sigma_{1},[] M_{2}, \Theta\right\rangle \lambda x . N$. with $\left\lceil\Sigma_{1},[] M_{2}, \Theta\right\rceil=\Phi$. By Lemma 3.3, $\left\langle\Sigma_{1},[] M_{2}, \Theta\right\rangle \lambda x . N$ is well-formed. By ind. hyp., $\vdash\left\langle\Sigma_{1}\right.$, $\Theta$, let rec $x^{\prime}$ be $M_{2}$ in [] $N\left[x^{\prime} / x\right] \Downarrow\left\langle\Sigma_{2}\right\rangle V$ with $\left\lceil\Sigma_{2}\right\rceil=\Psi^{\prime}$.

- The cases of Error $\beta$ and Letrec are immediate by induction.

- The case of Variable. Suppose we deduce $\langle\Psi\rangle x \Downarrow\langle\Phi[x \mapsto V]\rangle V$ from $\langle\Psi[x \mapsto \bullet]\rangle \Psi(x) \Downarrow\langle\Phi\rangle V$. Suppose $\lceil\Sigma\rceil=\Psi$ and $\langle\Sigma\rangle x$ is well-formed. There are three possible cases:

(i) When $\Psi(x)=\bullet$ and $\Sigma=\Sigma_{1}$, let rec $D, D_{x^{\prime}}$ in $E\left[x^{\prime}\right], \Sigma_{2}$ with $x \in D_{x^{\prime}}$. Then we deduce $\vdash\langle\Sigma\rangle x \Downarrow\langle\Sigma\rangle \bullet$ by Err $_{\text {var }}$.

(ii) When $\Psi(x)=N$ and $\Sigma=\Sigma_{1}$, let rec $x$ be $N, D$ in [], $\Sigma_{2}$. By ind. hyp. and Lemmas 3.4 and $3.5, \vdash\left\langle\Sigma_{1}\right.$, let rec $x$ be [], $D$ in $\left.\Sigma_{2}[x]\right\rangle N \Downarrow$ $\left\langle\Sigma_{1}^{\prime}\right.$, let rec $x$ be [], $D^{\prime}$ in $\left.\Sigma_{2}[x], \Theta\right\rangle V$ and $\left[\Sigma_{1}^{\prime}\right.$, let rec $x$ be [], $D^{\prime}$ in $\left.\Sigma_{2}[x], \Theta\right\rceil$ is the restriction of $\Phi$ to $L B V\left(\Sigma_{1}^{\prime}\right.$, let rec $x$ be [], $D^{\prime}$ in $\left.\Sigma_{2}[x], \Theta\right)$. Hence by Var we deduce $\vdash\left\langle\Sigma_{1}\right.$, let rec $x$ be $N, D$ in []$\left., \Sigma\right\rangle x \Downarrow\left\langle\Sigma_{1}^{\prime}\right.$, let rec $\bar{\Theta}, x$ be $V, D^{\prime}$ in [], $\left.\Sigma_{2}\right\rangle V$ and $\left[\Sigma_{1}^{\prime}\right.$, let $\operatorname{rec} \bar{\Theta}, x$ be $V, D^{\prime}$ in []$\left., \Sigma_{2}\right\rceil=\Phi[x \mapsto V]$.

(iii) The case in which $\Psi(x)=N$ and $\Sigma=\Sigma_{1}$, let rec $x$ be $N, D, D_{x^{\prime}}$ in $E\left[x^{\prime}\right], \Sigma_{2}$ is similar to the above case, except that we use $V_{a r}$ env instead of Var.

We prove the reduction semantics correct with respect to the natural semantics by proving three auxiliary results in Lemmas 3.6 and 3.7 and Corollary 3.1, which respectively correspond to Lemmas $2.8,2.7$ and 2.6 for the acyclic case.

We say a reduction sequence $M \rightarrow^{n} N$ is autonomous if either $n=0$ or the last step is reduced by rules other than assoc or $a s s o c_{\text {env }}$. These two rules have particular behaviour in that they flatten nested letrec's on request outside. We will restrict the use of the two rules by requiring a reduction sequence to be autonomous. We write $M \mapsto n$ to denote that $M$ reduces into $N$ in $n$-steps and the reduction sequence is autonomous. We may omit the suffix $n$ when it is irrelevant.

\section{Lemma 3.6}

The following two conditions hold:

(1) For any $\Theta, x, M, D$ and $E$ such that $\Theta$ [let rec $x$ be $M, D$ in $[E[x]]]$ is a program and $x$ is not in $L B V(E), \Theta[$ let rec $x$ be $M, D$ in $[E[x]]] \leftrightarrow^{n}$ $\Theta^{\prime}\left[\right.$ let rec $x$ be $A, D^{\prime}$ in $\left.E[x]\right]$ iff $\Theta[$ let rec $x$ be $\bullet, D$ in $M] \rightarrow^{n} \Theta^{\prime}\left[\right.$ let rec $x$ be $\bullet, D^{\prime}$ in $\left.A\right]$.

(2) For any $\Theta, D\left[x_{1}, x_{m}\right], M, D$ and $E$ such that $\Theta$ [let rec $D\left[x_{1}, x_{m}\right], x_{m}$ be $M, D$ in $\left.E\left[x_{1}\right]\right]$ is a program and $x_{1}$ is not in $\operatorname{LBV}(E)$ and $\operatorname{LBV}\left(D\left[x_{1}, x_{m}\right]\right)=\left\{x_{1}, \ldots, x_{m-1}\right\}$, $\Theta\left[\right.$ let rec $D\left[x_{1}, x_{m}\right], x_{m}$ be $M, D$ in $\left.E\left[x_{1}\right]\right] \leftrightarrow^{n} \Theta^{\prime}\left[\right.$ let rec $D\left[x_{1}, x_{m}\right], x_{m}$ be $A, D^{\prime}$ in $\left.E\left[x_{1}\right]\right]$ iff $\Theta\left[\right.$ let rec $x_{1}$ be $\bullet, \ldots, x_{m}$ be $\bullet, D$ in $\left.M\right] \rightarrow^{n} \Theta^{\prime}\left[\right.$ let rec $x_{1}$ be $\bullet, \ldots$, $x_{m}$ be $\bullet, D^{\prime}$ in $\left.A\right]$.

\section{Proof}

First we remark that the autonomy condition uniquely determines $n$ in the if case of both the conditions. We prove by simultaneous induction on the length of the reductions with case analysis on the possible reductions. 
- The case in which $M$ is an answer is obvious.

- The case in which $M$ reduces independent of the context is immediate by induction.

- The case in which $M=E^{\prime}\left[x^{\prime}\right]$ and $\Theta=\Theta_{1}$, let rec $x^{\prime}$ be $N, D_{1}$ in [], $\Theta_{2}$. We only prove the if case in (1). The other cases are similar. Suppose we have

$\Theta_{1}$ [let rec $x^{\prime}$ be $N, D_{1}$ in $\Theta_{2}$ [let rec $x$ be $E^{\prime}\left[x^{\prime}\right], D$ in $\left.[E[x]]\right]$

$\mapsto^{n_{1}} \Theta_{1}^{\prime}$ [let rec $x^{\prime}$ be $\Theta_{3}[V], D_{1}^{\prime}$ in $\Theta_{2}$ [let rec $x$ be $E^{\prime}\left[x^{\prime}\right], D$ in $\left.\left.[E[x]]\right]\right]$

$\rightarrow^{n_{2}} \Theta_{1}^{\prime}$ [let rec $x^{\prime}$ be $V, \overline{\Theta_{3}}, D_{1}^{\prime}$ in $\Theta_{2}$ [let rec $x$ be $E^{\prime}\left[x^{\prime}\right], D$ in $\left.\left.[E[x]]\right]\right]$

$\rightarrow \Theta_{1}^{\prime}\left[\right.$ let rec $x^{\prime}$ be $V, \overline{\Theta_{3}}, D_{1}^{\prime}$ in $\Theta_{2}\left[\right.$ let rec $x$ be $E^{\prime}[V], D$ in $\left.\left.[E[x]]\right]\right]$

$\mapsto^{n_{3}} \Theta^{\prime}$ [let rec $x$ be $A, D^{\prime}$ in $\left.E[x]\right]$

By ind. hyp., $\Theta_{1}$ [let rec $x^{\prime}$ be $\bullet, D_{1}$ in $\left.N\right] \rightarrow^{n_{1}} \Theta_{1}^{\prime}\left[\right.$ let rec $x^{\prime}$ be $\bullet, D_{1}^{\prime}$ in $\left.\Theta_{3}[V]\right]$.

Hence we have

$\Theta_{1}\left[\right.$ let rec $x^{\prime}$ be $N, D_{1}$ in $\Theta_{2}$ let rec $x$ be $\bullet, D$ in $\left.\left.E^{\prime}\left[x^{\prime}\right]\right]\right]$

$\leftrightarrow^{n_{1}} \Theta_{1}^{\prime}$ [let rec $x^{\prime}$ be $\Theta_{3}[V], D_{1}^{\prime}$ in $\Theta_{2}$ [let rec $x$ be $\bullet, D$ in $\left.\left.E^{\prime}\left[x^{\prime}\right]\right]\right]$ by ind. hyp.

$\rightarrow{ }^{n_{2}} \Theta_{1}^{\prime}\left[\right.$ let rec $x^{\prime}$ be $V, \overline{\Theta_{3}}, D_{1}^{\prime}$ in $\Theta_{2}$ [let rec $x$ be $\bullet, D$ in $\left.\left.E^{\prime}\left[x^{\prime}\right]\right]\right]$ by assoc

$\rightarrow \Theta_{1}^{\prime}$ [let rec $x^{\prime}$ be $V, \overline{\Theta_{3}}, D_{1}^{\prime}$ in $\Theta_{2}$ [let rec $x$ be $\bullet, D$ in $\left.\left.E^{\prime}[V]\right]\right]$ by deref

$\rightarrow^{n_{3}} \Theta^{\prime}$ [let rec $x$ be $\bullet, D^{\prime}$ in $\left.A\right]$ by ind. hyp.

- The cases in which $M=E^{\prime}[x]$ in (1) and in which $M=E^{\prime}\left[x_{i}\right]$ for some $i$ in $1, \ldots, m$ in (2) are immediate by induction.

- The case in which $M=E^{\prime}\left[x^{\prime}\right]$ and $x^{\prime}$ is in $L B V(D)$ for the if case in (1).

Suppose we have

$\Theta$ [let rec $x$ be $E^{\prime}\left[x^{\prime}\right], x^{\prime}$ be $N, D_{1}$ in $\left.E[x]\right]$

$\mapsto^{n_{1}} \Theta_{1}$ [let rec $x$ be $E^{\prime}\left[x^{\prime}\right], x^{\prime}$ be $\Theta_{2}[V], D_{1}^{\prime}$ in $\left.E[x]\right]$

$\rightarrow^{n_{2}} \Theta_{1}$ [let rec $x$ be $E^{\prime}\left[x^{\prime}\right], \overline{\Theta_{2}}, x^{\prime}$ be $V, D_{1}^{\prime}$ in $\left.E[x]\right]$

$\rightarrow \Theta_{1}\left[\right.$ let rec $x$ be $E^{\prime}[V], \overline{\Theta_{2}}, x^{\prime}$ be $V, D_{1}^{\prime}$ in $\left.E[x]\right]$

$\leftrightarrow^{n_{3}} \Theta^{\prime}\left[\right.$ let rec $x$ be $A, D^{\prime}$ in $\left.E[x]\right]$

By ind. hyp., $\Theta$ [let rec $x$ be $\bullet, x^{\prime}$ be $\bullet, D_{1}$ in $\left.N\right] \rightarrow^{n_{1}} \Theta_{1}$ [let rec $x$ be $\bullet, x^{\prime}$ be $\bullet$, $D_{1}^{\prime}$ in $\left.\Theta_{2}[V]\right]$.

Hence we have

$\Theta\left[\right.$ let rec $x$ be $\bullet, x^{\prime}$ be $N, D_{1}$ in $\left.E^{\prime}\left[x^{\prime}\right]\right]$

$\mapsto^{n_{1}} \Theta_{1}$ [let rec $x$ be $\bullet, x^{\prime}$ be $\Theta_{2}[V], D_{1}^{\prime}$ in $\left.E^{\prime}\left[x^{\prime}\right]\right]$ by ind. hyp.

$\rightarrow^{n_{2}} \Theta_{1}\left[\right.$ let rec $x$ be $\bullet, \overline{\Theta_{2}}, x^{\prime}$ be $V, D_{1}^{\prime}$ in $\left.E^{\prime}\left[x^{\prime}\right]\right]$ by assoc

$\rightarrow \Theta_{1}\left[\right.$ let rec $x$ be $\bullet, \overline{\Theta_{2}}, x^{\prime}$ be $V, D_{1}^{\prime}$ in $\left.E^{\prime}[V]\right]$ by deref

$\rightarrow \Theta^{\prime}\left[\right.$ let rec $D^{\prime}$ in $\left.A\right]$ by ind. hyp.

- The cases in which $M=E^{\prime}\left[x^{\prime}\right]$ and $x^{\prime}$ is in $L B V(D)$ for the only if case in (1) and the if and only if cases in (2) are similar to the above case.

\section{Corollary 3.1}

For any $\Theta, E$ and $x$ such that $\Theta[E[x]]$ is a program and $x$ is not in $L B V(E)$, if $\Theta[E[x]] \rightarrow^{n} \Theta^{\prime}[E[V]]$, then for any $E^{\prime}$ such that $\Theta\left[E^{\prime}[x]\right]$ is a program and $x$ is not in $L B V\left(E^{\prime}\right), \Theta\left[E^{\prime}[x]\right] \rightarrow^{n} \Theta^{\prime}\left[E^{\prime}[V]\right]$.

We adapt the definition of rooted reductions in an obvious way by replacing let with let rec. A reduction $M \rightarrow M^{\prime}$ is $\beta_{\text {need }}$-rooted with argument $N$ if $M=$ $\Theta\left[\left(\lambda x . N^{\prime}\right) N\right]$ and $M^{\prime}=\Theta\left[\right.$ let rec $x$ be $N$ in $\left.N^{\prime}\right]$. A reduction sequence $M \rightarrow M^{\prime}$ 
preserves a $\beta_{\text {need }}$-root with argument $N$ if none of the (one-step) reductions in the sequence is $\beta_{\text {need }}$-rooted with argument $N$. The following lemma is proved similarly as Lemma 2.7.

\section{Lemma 3.7}

For any $\Theta, M$ and $N$ such that $\Theta[M N]$ is a program, if $\Theta[M N] \rightarrow^{n} \Theta^{\prime}[V N]$ and the reduction sequence preserves a $\beta_{\text {need }}$-root with argument $N$, then $\Theta[M] \rightarrow^{n^{\prime}} \Theta^{\prime}[V]$ with $n^{\prime} \leqslant n$.

Now we are ready to prove the reduction semantics correct with respect to the natural semantics.

\section{Proposition 3.3}

For any program $M$, if $M \rightarrow A$, then there exist $\Theta$ and $V$ such that $\Theta[V]$ and $A$ belong to the same $\alpha$-equivalence class and \langle\rangle $M \Downarrow\langle\lceil\Theta\rceil\rangle V$.

Proof

Without loss of generality, we assume $\Theta[V]$ and $A$ are syntactically identical. We prove by induction on the length of the reductions of $M$. Let $M=\Theta^{\prime}\left[M^{\prime}\right]$ with $M^{\prime} \neq$ let rec $D$ in $N$. We perform case analysis on $M^{\prime}$.

- The case of an answer is obvious.

- Suppose $M=M_{1} M_{2}$ and we have

$$
\Theta^{\prime}\left[M_{1} M_{2}\right] \rightarrow \Theta_{1}\left[(\lambda x . N) M_{2}\right] \rightarrow \Theta_{1}\left[\text { let rec } x \text { be } M_{2} \text { in } N\right] \rightarrow \Theta[V]
$$

By Lemma 3.7 and ind. hyp., \langle\rangle$\Theta^{\prime}\left[M_{1}\right] \Downarrow\left\langle\left\lceil\Theta_{1}\right\rceil\right\rangle \lambda x . N$. By ind. hyp.,

\langle\rangle$\Theta_{1}\left[\right.$ let rec $x$ be $M_{2}$ in $\left.N\right] \Downarrow\langle\lceil\Theta\rceil\rangle V$. Thus we deduce \langle\rangle$\Theta^{\prime}\left[M_{1} M_{2}\right] \Downarrow\langle\lceil\Theta\rceil\rangle V$.

- The case in which $M=M_{1} M_{2}$ and $M_{1}$ reduces to $\bullet$ is similar to the above case.

- Suppose $M=x$ and $\Theta=\Theta_{1}$, let rec $x$ be $N, D$ in [], $\Theta_{2}$ and we have

$$
\begin{aligned}
& \Theta_{1}\left[\text { let rec } x \text { be } N, D \text { in } \Theta_{2}[x]\right] \\
& \rightarrow^{n} \Theta_{1}^{\prime}\left[\text { let rec } x \text { be } \Theta_{3}[V], D_{1} \text { in } \Theta_{2}[x]\right] \\
& \left.\rightarrow \Theta_{1}^{\prime} \text { [let rec } x \text { be } V, \bar{\Theta}_{3}, D_{1} \text { in } \Theta_{2}[x]\right] \\
& \left.\rightarrow \Theta_{1}^{\prime} \text { [let rec } x \text { be } V, \overline{\Theta_{3}}, D_{1} \text { in } \Theta_{2}[V]\right]
\end{aligned}
$$

By Lemma 3.6, $\Theta_{1}[$ let rec $x$ be $\bullet, D$ in $N] \rightarrow^{n} \Theta_{1}^{\prime}$ [let rec $x$ be $\bullet, D_{1}$ in $\left.\Theta_{3}[V]\right]$. By ind. hyp., \langle\rangle$\Theta_{1}$ [let rec $x$ be $\bullet, D$ in $\left.N\right] \Downarrow\left\langle\left\lceil\Theta_{1}^{\prime}\right.\right.$, let rec $x$ be $\bullet, D_{1}$ in [], $\left.\left.\Theta_{3}\right\rceil\right\rangle V$. By Lemma 3.5, $\left\langle\left\lceil\Theta_{1}\right.\right.$, let rec $x$ be $\bullet, D$ in [], $\left.\left.\Theta_{2}\right\rceil\right\rangle N \Downarrow\left\langle\left\lceil\Theta_{1}^{\prime}\right.\right.$, let rec $x$ be $\bullet, D_{1}$ in [], $\left.\left.\Theta_{3}, \Theta_{2}\right\rceil\right\rangle V$. Thus we deduce \langle\rangle$\Theta_{1}$ [let rec $x$ be $N, D$ in $\left.\Theta_{2}[x]\right] \Downarrow$ $\left\langle\left\lceil\Theta_{1}^{\prime}\right.\right.$, let rec $x$ be $V, \overline{\Theta_{3}}, D_{1}$ in []$\left.\left., \Theta_{2}\right\rceil\right\rangle V$.

Collecting all propositions together, we prove equivalence of the two semantics.

Theorem 3.1

For any program $M$, the following two conditions hold:

(1) if $M \rightarrow A$, then there exist $\Theta$ and $V$ such that $\Theta[V]$ and $A$ belong to the same $\alpha$-equivalence class and \langle\rangle $M \Downarrow\langle\lceil\Theta\rceil\rangle V$;

(2) if \langle\rangle $M \Downarrow\langle\Psi\rangle V$, then $M \rightarrow \Theta[V]$ where $\lceil\Theta\rceil=\Psi$. 
Proof

(1) By Proposition 3.3.

(2) By Proposition 3.2 and Lemma 3.4, $\vdash\langle\rangle M \Downarrow\langle\Theta\rangle V$ with $\lceil\Theta\rceil=\Psi$. By Proposition 3.1, $M \rightarrow \Theta[V]$.

\subsection{Adequacy}

In this subsection we state that the natural semantics is adequate using a denotational semantics in the style of Launchbury (1993). Fortunately we can adapt his proof strategy with minor modifications. Below we only recall the necessary definitions from his paper to state main propositions; we refer the details to his original paper and the extended version of ours (Nakata \& Hasegawa 2009).

We define the denotational semantics for pure expressions of $\lambda_{\text {letrec. An expression }}$ is pure if it does not contain black holes. The denotational semantics models functions by a lifted function space (Abramsky \& Ong 1993). We represent lifting using $F n$ and projection using $\downarrow_{F n}$ (written as a postfix operator). Let Values be some appropriate domain containing at least a lifted version of its own function space. Environments, ranged over by $\rho$, are functions from Vars to Values, where

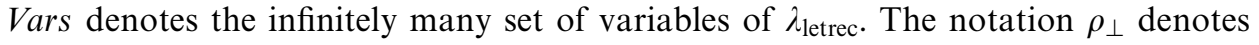
an 'initial' environment which maps all variables to $\perp$.

The semantic functions $\llbracket M \rrbracket_{\rho}$ and $\{D\}_{\rho}$ respectively give meanings to the expression $M$ and the bindings $D$ under the environment $\rho$. The former returns an element from Value and the latter an environment. They are defined by mutual recursion as follows:

$$
\begin{aligned}
& \llbracket \lambda x . M \rrbracket_{\rho}=F n\left(\lambda v \cdot \llbracket M \rrbracket_{\rho \sqcup\{x \mapsto v\}}\right) \\
& \llbracket M N \rrbracket_{\rho}=\left(\llbracket M \rrbracket_{\rho}\right) \downarrow_{F n}\left(\llbracket N \rrbracket_{\rho}\right) \\
& \llbracket x \rrbracket_{\rho}=\rho(x) \\
& \llbracket \text { let rec } x_{1} \text { be } M_{1}, \ldots, x_{n} \text { be } M_{n} \text { in } N \rrbracket_{\rho}=\llbracket N \rrbracket_{\left\{x_{1} \text { be } M_{1}, \ldots, x_{n} \text { be } M_{n}\right\}_{\rho}} \\
& \left\{x_{1} \text { be } M_{1}, \ldots, x_{n} \text { be } M_{n}\right\}_{\rho}=\mu \rho^{\prime} . \rho \sqcup\left\{x_{1} \mapsto \llbracket M_{1} \rrbracket_{\rho^{\prime}}, \ldots, x_{n} \mapsto \llbracket M_{n} \rrbracket_{\rho^{\prime}}\right\}
\end{aligned}
$$

where $\mu$ denotes the least fixed point operator; $\{D\}_{\rho}$ is defined only when $\rho$ is consistent with $D$; i.e. if $\rho$ and $D$ bind the same variable, then they map the variable to values for which an upper bound exists. The semantic function for heaps is defined in the same way as that for bindings by identifying a heap with an unordered sequence of bindings.

Proposition 3.4 states that derivations preserve non-bottom meanings of pure expressions; Proposition 3.5 states that a pure expression evaluates to an abstraction if and only if its meaning is a non-bottom element. Since the natural semantics is deterministic, we can deduce that if a pure expression evaluates to a black hole, then its meaning is a bottom element.

\section{Proposition 3.4}

For any pure program $M$, if \langle\rangle $M \Downarrow\langle\Psi\rangle \lambda x . N$, then $\llbracket M \rrbracket_{\rho_{\perp}}=\llbracket \lambda x . N \rrbracket_{\{\Psi\}_{\rho_{\perp}}}$. 
Proposition 3.5

For any pure program $M, \llbracket M \rrbracket_{\rho_{\perp}} \neq \perp$ iff \langle\rangle $M \Downarrow\langle\Psi\rangle \lambda x . N$.

\section{Related work}

Our work builds on previous work by Launchbury (1993), Sestoft (1997), Ariola and Felleisen (1997) and Maraist et al. (1998). The reduction semantics present in the paper are mostly identical to those of Ariola and Felleisen. As to the natural semantics for $\lambda_{\text {let }}$, we revised that of Maraist et al. by correctly enforcing variable hygiene in the style of Sestoft and by explicitly introducing an inference rule for the let construct. As to the natural semantics for $\lambda_{\text {letrec }}$, we revised that of Sestoft by eliminating the precompilation step. Adequacy of the natural semantics for $\lambda_{\text {let }}$ is ascribed to its correspondence with the reduction semantics, which is proved equivalent to call-by-name by Ariola and Felleisen. In turn we showed adequacy of the natural semantics for $\lambda_{\text {letrec }}$ by adapting Launchbury's denotational argument. Adequacy of the reduction semantics for $\lambda_{\text {letrec }}$ is then ascribed to its correspondence with the natural semantics; to the best of our knowledge, this fact has not been shown so far. In the above-discussed sense, our work extends those previous work.

There are several lines of work which considers other styles of formalization of call-by-need in the presence or absence of recursion. Below we review some of them. The reader may be interested in the concluding remarks of Maraist et al. (1998), where they have discussed the reduction semantics in relation to other systems.

Recent work by Garcia et al. (2009) proposed an abstract machine for the let-free formulation of the acyclic calculus $\lambda_{\text {let }}$, which is proved equivalent to the reduction semantics of Ariola and Felleisen (1997). They also presented a simulation of the machine by a call-by-value lambda calculus extended with delimited control operators. While developed independently, their abstract machine, in particular the refined one, and our instrumented natural semantics bear similarities in that both manipulate sequenced evaluation contexts while retaining the structural knowledge of a term that has been discovered. More thorough comparison might suggest a means of simulating the cyclic calculus $\lambda_{\text {letrec }}$ using delimited control. This is one direction for future work.

Sestoft revised the natural semantics of Launchbury by enforcing variable hygiene correctly and changing the $\alpha$-renaming strategy (Sestoft 1997). He derived an abstract machine for call-by-need from the revised semantics. The machine has a small-step semantics and uses global heaps to implement sharing of evaluation. Starting from a simple machine, he refines it to a more efficient machine in several steps. The machine is proved equivalent to his natural semantics. As discussed earlier, the natural semantics for $\lambda_{\text {letrec }}$ is strongly inspired by his semantics.

Okasaki et al. (1994) proposed a transformation of call-by-need $\lambda$ terms, in the absence of recursion, into continuation-passing style, which is proved equivalent to a call-by-need continuation semantics. Sharing of evaluation is implemented by ML-style references, which resemble global heaps.

Ariola and Klop (1994) and Ariola and Blom (1997) studied equational theories of cyclic lambda calculi by means of cyclic lambda graphs. The former observed 
that having non-restricted substitution leads to non-confluence and proposed a restriction on substitution to recover confluence. The latter proposed a relaxed notion of confluence which holds in the presence of non-restricted substitution. In Ariola and Blom (1997) a calculus supporting sharing is considered, but a reduction strategy for the calculus is not studied.

Danvy (2008) has advocated the use of abstract machines as a 'natural meeting ground' of various functional implementations of operational semantics, especially the small-step reduction semantics and big-step natural semantics. In a large perspective, our work presented here can be thought as making an analogous case for a destructive, non-functional setting, in which circularly shared computation contributes significant complexities.

\section{Conclusion}

We have presented natural semantics for acyclic and cyclic call-by-need lambda calculi, which are proved equivalent to the reduction semantics given by Ariola and Felleisen (1997). We observed differences of the two styles of formalization in the treatment of when to reorganize the heap structure and how to focus redexes. The proof uses instrumented natural semantics as mediatory semantics of the two, in order to bridge these differences by making heap reorganization and redex focusing explicit.

This work is initially motivated to study lazy evaluation strategies for recursive records in terms of the reduction semantics as well as the natural semantics. The extended paper (Nakata \& Hasegawa 2009) considers an extension of $\lambda_{\text {letrec }}$ with pairs and a call-by-value variant with lazy letrec. The variant can emulate the delay and force operators as provided in Scheme (Sperber et al. 2009), or OCaml's equivalent lazy and force (Leroy et al. 2008), by let rec $x$ be $M$ in $\lambda x^{\prime} . x$ for delay $(M)$ and $M(\lambda x . x)$ for force $(M)$. For instance, the initialization graphs of Syme (2005), which underlie the object initialization strategy of F\# (Syme \& Margetson 2008), fit in the call-by-value variant extended with $n$-tuples, or records.

\section{Acknowledgments}

We thank the anonymous referees for their careful reviewing and Matthias Felleisen for his editorial support. Keiko Nakata has been supported by the Estonian Science Foundation grant no. 6940 and the ERDF cofunded project EXCS, the Estonian Centre of Excellence in Computer Science. Masahito Hasegawa has partly been supported by the Grant-in-Aid for Scientific Research (C) 20500010.

\section{References}

Abramsky, S. \& Ong, C.-H. L. (1993) Full abstraction in the lazy lambda calculus, Inf. Comput., 105 (2): 159-267.

Ariola, Z. \& Felleisen, M. (1997) The call-by-need lambda calculus. J. Funct. Program., 7 (3): 265-301. 
Ariola, Z. M. \& Blom, S. (1997) Cyclic lambda calculi. In Proc. Theoretical Aspects of Computer Software. Abadi, M. and Ito, T. (eds), Lecture Notes in Computer Science, vol. 1281. Springer, Berlin/Heidelberg, pp. 77-106.

Ariola, Z. M. \& Klop, J. W. (1994) Cyclic lambda graph rewriting. In Proceedings of the Symposium on Logic in Computer Science, Abramsky, S. (ed), Paris, France, pp. 416-425.

Danvy, O. (2008) Defunctionalized interpreters for programming languages. In Proceedings of the International Conference on Functional Programming, James Hook and Peter Thiemann (eds), Victoria, BC, Canada, ACM Press.

Garcia, R., Lumsdaine, A \& Sabry, A. (2009) Lazy evaluation and delimited control. In Proceedings of the ACM SIGPLAN-SIGACT Symposium on the Principles of Programming Languages, Zhong Shao and Benjamin C. Pierce (eds), Savannah, GA, USA. ACM Press.

Launchbury, J. (1993) A natural semantics for lazy evaluation. In Proceedings of the ACM SIGPLAN-SIGACT Symposium on the Principles of Programming Languages, Charleston, $\mathrm{SC}$, USA.

Leroy, X., Doligez, D., Garrigue, J., Rémy, D. \& Vouillon, J. (2008) The Objective Caml system, release 3.11 [online]. Available at: http://caml.inria.fr/ (Accessed 29 July 2009).

Maraist, J., Odersky, M. \& Wadler, P. (1998) A call-by-need lambda calculus, J. Funct. Program., 8 (3): 275-317.

Nakata, K. \& Hasegawa, M. (2009) Small-step and big-step semantics for call-by-need (extended version). ArXiv:0907.4640v1 [cs.PL].

Okasaki, C., Lee, P. \& Tarditi, D. (1994) Call-by-need and continuation-passing style, LISP Symbol. Comput., 7 (1): 57-82.

Plotkin, G. (1975) Call-by-name, Call-by-value and the $\lambda$-Calculus, Theoret. Comp. Sci., 1 (2): $125-159$.

Sestoft, P. (1997) Deriving a lazy abstract machine, J. Funct. Program., 7 (3): 231-264.

Sperber, M., Dybvig, R. K., Flatt, M. \& Straaten, A. V. (2009) Revised ${ }^{6}$ Report on the Algorithmic Language Scheme. J. Funct. Program., 19 (S1): 1-301.

Syme, D. (2005) Initializing mutually referential abstract objects: The value recursion challenge. In Proceedings of the Workshop on ML, Electr. Notes Theor. Comput. Sci., Tallinn, Estonia, 148 (2): 3-25.

Syme, D. \& Margetson, J. (2008) The F\# programming language [online]. Available at: http://research.microsoft.com/en-us/um/people/curtisvv/fsharp_default.aspx (Accessed 29 July 2009).

Wright, A. K. \& Felleisen, M. (1994) A syntactic approach to type soundness. Information and Computation, 115 (1): 38-94. 